\title{
Probing accretion of ambient cloud material into the Taurus B211/B213 filament
}

\author{
Y. Shimajiri ${ }^{1}$, Ph. André ${ }^{1}$, P. Palmeirim² ${ }^{2}$ D. Arzoumanian ${ }^{3}$, A. Bracco ${ }^{4}$, V. Könyves ${ }^{1}$, E. Ntormousi ${ }^{5}$, and B. Ladjelate ${ }^{6}$ \\ ${ }^{1}$ Laboratoire AIM, CEA/DRF-CNRS-Université Paris Diderot, IRFU/Département d'Astrophysique, C.E. Saclay, \\ Orme des Merisiers, 91191 Gif-sur-Yvette, France \\ e-mail: Yoshito.Shimajiri@cea.fr \\ ${ }^{2}$ Instituto de Astrofísica e Ciências do Espaço, Universidade do Porto, CAUP, Rua das Estrelas, 4150-762 Porto, Portugal \\ ${ }^{3}$ Department of Physics, Graduate School of Science, Nagoya University, Nagoya 464-8602, Japan \\ ${ }^{4}$ Nordita, KTH Royal Institute of Technology and Stockholm University, Roslagstullsbacken 23, 10691 Stockholm, Sweden \\ ${ }^{5}$ Foundation for Research and Technology (FORTH), Nikolaou Plastira 100, Vassilika Vouton GR - 711 10, Heraklion, Crete, \\ Greece \\ ${ }^{6}$ Instituto Radioastronomia Milimétrica, Av. Divina Pastora 7, Nucleo Central, 18012 Granada, Spain
}

Received 8 October 2018 / Accepted 7 November 2018

\begin{abstract}
Context. Herschel observations have emphasized the role of molecular filaments in star formation. However, the origin and evolution of these filaments are not yet well understood, partly because of the lack of kinematic information.

Aims. We confirm from a kinematic viewpoint that the Taurus B211/B213 filament is accreting background cloud material, and we investigate the potential influence of large-scale external effects on the formation of the filament.

Methods. To examine whether the B211/B213 filament is accreting background gas because of its gravitational potential, we produced a toy accretion model and compared its predictions to the velocity patterns observed in ${ }^{12} \mathrm{CO}(1-0)$ and ${ }^{13} \mathrm{CO}(1-0)$. We also examined the spatial distributions of $\mathrm{H} \alpha$, Planck $857 \mathrm{GHz}$ dust continuum, and $\mathrm{HI}$ emission to search for evidence of large-scale external effects. Results. We estimate that the depth of the Taurus cloud around the B211/B213 filament is $\sim 0.3-0.7 \mathrm{pc}$ under the assumption that the density of the gas is the same as the critical density of ${ }^{13} \mathrm{CO}(1-0)$. Compared to a linear extent of $>10 \mathrm{pc}$ in the plane of the sky, this suggests that the 3D morphology of the cloud surrounding the B211/B213 filament is sheet-like. Position-velocity $(P V)$ diagrams observed in ${ }^{12} \mathrm{CO}(1-0)$ and ${ }^{13} \mathrm{CO}(1-0)$ perpendicular to the filament axis show that the emission from the gas surrounding B211/B213 is redshifted to the northeast of the filament and blueshifted to the southwest, and that the velocities of both components approach the velocity of the B211/B213 filament as the line of sight approaches the crest of the filament. The $P V$ diagrams predicted by our accretion model are in good agreement with the observed ${ }^{12} \mathrm{CO}(1-0)$ and ${ }^{13} \mathrm{CO}(1-0) P V$ diagrams, supporting the previously proposed scenario of mass accretion into the filament. Moreover, inspection of the spatial distribution of the $\mathrm{H} \alpha$ and Planck $857 \mathrm{GHz}$ emission in the Taurus-California-Perseus region on scales up to $>200$ pc suggests that the B211/B213 filament may have formed as a result of an expanding supershell generated by the Per OB2 association.

Conclusions. Based on these results, we propose a scenario in which the B211/B213 filament was initially formed by large-scale compression of $\mathrm{HI}$ gas and is now growing in mass by gravitationally accreting molecular gas of the ambient cloud.
\end{abstract}

Key words. ISM: clouds - ISM: kinematics and dynamics

\section{Introduction}

The observations of the Herschel Gould Belt survey (HGBS) have revealed that parsec-scale filaments are ubiquitous in molecular clouds and emphasized their importance for solartype star formation (e.g., André et al. 2010; Men'shchikov et al. 2010; Arzoumanian et al. 2011; Palmeirim et al. 2013). In particular, most Herschel prestellar cores are found to lie in dense (thermally supercritical) filaments, suggesting that cores generally form by filament fragmentation (e.g., Könyves et al. 2015; Marsh et al. 2016; Benedettini et al. 2018). Molecular line observations of the velocity field around cores and filaments further support this view (Tafalla \& Hacar 2015). Based on the HGBS results, André et al. (2014) proposed a filament paradigm for star formation, whereby large-scale compression of interstellar material in supersonic flows generates a quasi-universal web of $\sim 0.1$-pc wide filaments in the cold interstellar medium (ISM) and then the denser filaments fragment into prestellar cores by gravitational instability. Recently, Shimajiri et al. (2017) found that the star formation efficiency in dense molecular gas $\left(A_{\mathrm{v}}>8\right)$, where filamentary structures dominate the mass budget, is remarkably uniform over a wide range of scales from 1-10 pc to >10 kpc (see also Gao \& Solomon 2004; Lada et al. 2010, 2012; Chen et al. 2015). Furthermore, Shimajiri et al. (2017) proposed that this common star formation efficiency in dense gas results from the microphysics of star formation in filaments (see also André et al. 2014). This result suggests the existence of a universal "star formation law" for converting dense molecular gas into stars along filaments. Therefore, unveiling how molecular filaments grow in mass and fragment is crucial to understanding star formation in filaments.

The B211/B213 filament system is located in the Taurus molecular cloud, which is one of the nearest star-forming regions ( $d \sim 140 \mathrm{pc}$; Elias 1978). Wide-field mapping observations in ${ }^{12} \mathrm{CO},{ }^{13} \mathrm{CO}, \mathrm{C}^{18} \mathrm{O}, \mathrm{N}_{2} \mathrm{H}^{+}$, and $\mathrm{SO}$ emission revealed a whole network of filamentary structures in the B211/B213 area 

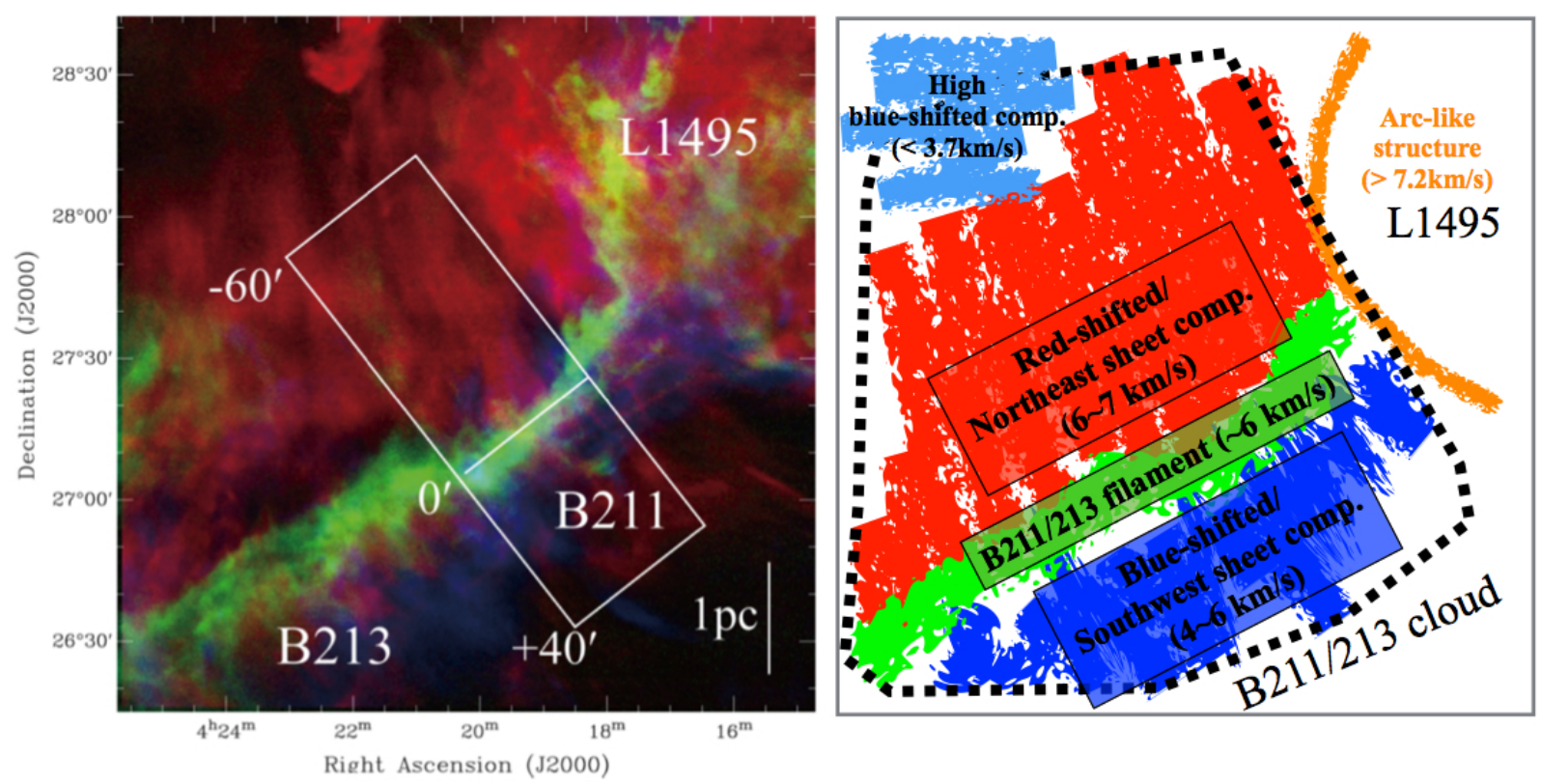

Fig. 1. Left panel: ${ }^{12} \mathrm{CO}(1-0)$ and ${ }^{13} \mathrm{CO}(1-0)$ emission observed toward the B211/B213 filament. Right panel: schematic picture of the velocity components. The ${ }^{12} \mathrm{CO}$ and ${ }^{13} \mathrm{CO}$ data are from Goldsmith et al. (2008). The left panel is adopted from Palmeirim et al. (2013). In the left panel the red color shows the distribution of the ${ }^{12} \mathrm{CO}(1-0)$ emission with a velocity range of $6.6-7.4 \mathrm{~km} \mathrm{~s}^{-1}$, the green color shows ${ }^{13} \mathrm{CO}(1-0)$ emission with a velocity range of 5.6-6.4 $\mathrm{km} \mathrm{s}^{-1}$, and the blue color shows ${ }^{12} \mathrm{CO}(1-0)$ emission with a velocity range of $4.2-5.5 \mathrm{~km} \mathrm{~s}$. The white box perpendicular to the filament axis shows the cut line for the position velocity diagrams shown in Fig. 6.

(Goldsmith et al. 2008; Hacar et al. 2013; Panopoulou et al. 2014; Tafalla \& Hacar 2015). Goldsmith et al. (2008) and Palmeirim et al. (2013) found that many low-density striations are elongated parallel to the magnetic field, and that blueshifted and redshifted components in both ${ }^{12} \mathrm{CO}(1-0)$ and ${ }^{13} \mathrm{CO}(1-0)$ emission are distributed southwest and northeast of the B211/B213 filament, as shown in Fig. 1. This morphology was suggestive of mass accretion along magnetic field lines into the B211/B213 filament. To quantify mass accretion, Palmeirim et al. (2013) assumed cylindrical geometry and used the observed mass per unit length $M_{\text {line }}$ to estimate the gravitational acceleration $\phi(R)=2 G M_{\text {line }} / R$ of a piece of gas in free-fall toward the filament (where $R$ and $G$ denote radius from filament center and the gravitational constant, respectively). The free-fall velocity of gas initially at rest at a cylindrical radius $R_{\text {init }} \sim 2$ pc was estimated to reach $1.1 \mathrm{~km} \mathrm{~s}^{-1}$ when the material reached the outer radius $R_{\text {out }} \sim 0.4 \mathrm{pc}$ of the B211/B213 filament. This estimation was consistent with the velocity observed in $\mathrm{CO}$, suggesting that the background gas accretes into the B211/B213 filament owing to the gravitational potential of the B211/B213 filament. However, the velocity structure was not investigated in detail. Investigation of the velocity structure is crucial to confirm this suggested scenario from the kinematic viewpoint. This is the topic of the present paper.

The paper is organized as follows: in Sect. 2 we describe the ${ }^{12} \mathrm{CO}(1-0)$ and ${ }^{13} \mathrm{CO}(1-0)$ data, as well as complementary $\mathrm{H} \alpha$, $857 \mathrm{GHz}$, and HI data. In Sect. 3 we estimate the optical depth of the ${ }^{12} \mathrm{CO}(1-0)$ line and present the ${ }^{12} \mathrm{CO}(1-0)$ and ${ }^{13} \mathrm{CO}(1-0)$ velocity structures observed in the B211/B213 cloud. In Sect. 4 we discuss the cloud structure, whether the surrounding material accretes onto the B211/B213 filament from the kinematic viewpoint, and whether the filament is formed by large-scale compression. In Sect. 5 we summarize our results.

\section{Observational data}

We used the ${ }^{12} \mathrm{CO}(1-0)$ and ${ }^{13} \mathrm{CO}(1-0)$ data obtained by Goldsmith et al. (2008) and Narayanan et al. (2008) with the
$14 \mathrm{~m}$ diameter millimeter-wave telescope of the Five College Radio Astronomy Observatory (FCRAO). The half-power beam width of the telescope was $45^{\prime \prime}$ for ${ }^{12} \mathrm{CO}(1-0)$ and $47^{\prime \prime}$ for ${ }^{13} \mathrm{CO}(1-0)$. We applied Gaussian spatial smoothing to improve the signal-to-noise ratio $(\mathrm{S} / \mathrm{N})$, resulting in an effective beam resolution of $\sim 76^{\prime \prime}$, corresponding to $\sim 0.05 \mathrm{pc}$ at a distance of $140 \mathrm{pc}$. The velocity resolution of the data is $0.26 \mathrm{~km} \mathrm{~s}^{-1}$ for ${ }^{12} \mathrm{CO}(1-0)$ and $0.27 \mathrm{~km} \mathrm{~s}^{-1}$ for ${ }^{13} \mathrm{CO}(1-0)$. The rms noise level is $0.1 \mathrm{~K}\left(T_{\mathrm{A}}^{*}\right)$ for ${ }^{12} \mathrm{CO}(1-0)$ and $0.05 \mathrm{~K}\left(T_{\mathrm{A}}^{*}\right)$ for ${ }^{13} \mathrm{CO}(1-0)$. As complementary observations of the Taurus cloud region and its large-scale environment, we also used the $\mathrm{H} \alpha$ data $^{1}$ of Finkbeiner (2003), as well as Planck $857 \mathrm{GHz}^{2}$ (Planck Collaboration I 2014) and HI data ${ }^{3}$ (Kalberla et al. 2017) from the archive.

\section{Analysis and results}

\section{1. ${ }^{12} \mathrm{CO}(1-0)$ and ${ }^{13} \mathrm{CO}(1-0)$ optical depths}

The optical depth of the ${ }^{12} \mathrm{CO}(1-0)$ line was estimated from the FCRAO ${ }^{12} \mathrm{CO}$ and ${ }^{13} \mathrm{CO}$ data. Assuming the same excitation temperature for the ${ }^{12} \mathrm{CO}(1-0)$ and ${ }^{13} \mathrm{CO}(1-0)$ lines, an isotopic ratio, $R_{\mathrm{i}}=62$ for ${ }^{12} \mathrm{C} /{ }^{13} \mathrm{C}$ (Langer \& Penzias 1993), and the same beam filling factor in both lines, we evaluated the optical depth of ${ }^{12} \mathrm{CO}(1-0)$ using the following equation:

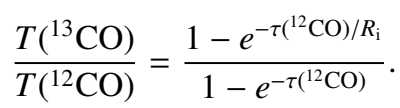

Here, $T\left({ }^{12} \mathrm{CO}\right)$ and $\tau\left({ }^{12} \mathrm{CO}\right)$ denote the peak intensity and the optical depth of ${ }^{12} \mathrm{CO}(1-0)$, respectively. While the ${ }^{12} \mathrm{CO}(1-0)$

\footnotetext{
1 https://faun.rc.fas.harvard.edu/dfink/skymaps/ halpha/data/v1_1/index.html

2 https://irsa.ipac.caltech.edu/data/Planck/release_ 1/all-sky-maps/previews/HFI_SkyMap_857_2048_R1.10_ survey_2_ZodiCorrected/

3 https://www.astro.uni-bonn.de/hisurvey/Allsky_ profiles/index.php
} 


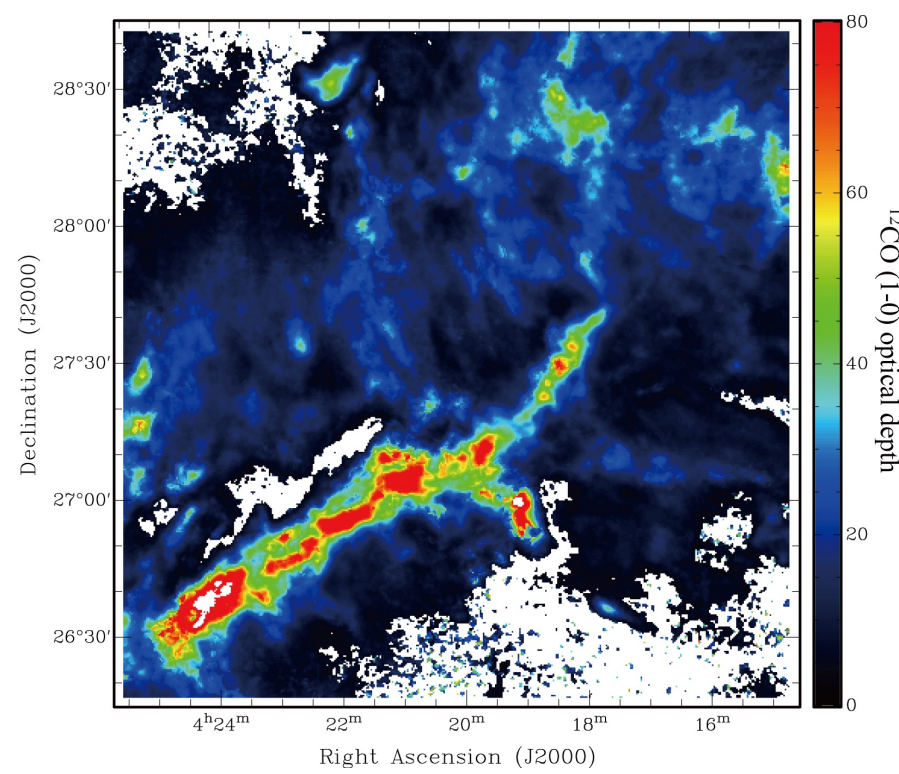

Fig. 2. Map of ${ }^{12} \mathrm{CO}(1-0)$ optical depth derived from the Goldsmith et al. (2008) ${ }^{12} \mathrm{CO}(1-0)$ and ${ }^{13} \mathrm{CO}(1-0)$ data.

emission preferentially traces the diffuse extended cloud, the ${ }^{13} \mathrm{CO}(1-0)$ emission traces the central B211/B213 filament (see Fig. 1). The typical inner width of the filaments observed with Herschel is $\sim 0.1 \mathrm{pc}$ (Arzoumanian et al. 2011, 2019; Palmeirim et al. 2013), which is larger than the 0.05 pc effective beam size of the FCRAO data. Thus, assuming the same beam filling factor in ${ }^{12} \mathrm{CO}(1-0)$ and ${ }^{13} \mathrm{CO}(1-0)$ is reasonable. Figure 2 displays the resulting map of the ${ }^{12} \mathrm{CO}(1-0)$ optical depth. The optical depth in this map ranges from $\sim 3$ to $\sim 300$, showing that the ${ }^{12} \mathrm{CO}(1-0)$ emission is optically thick. In particular, the ${ }^{12} \mathrm{CO}(1-0)$ optical depth toward the B211/B213 filament itself $\left(\tau\left({ }^{12} \mathrm{CO}\right) \sim 100\right)$ is much larger than that found for the surrounding lower density material $\left(\tau\left({ }^{12} \mathrm{CO}\right) \sim 20\right)$.

\section{2. ${ }^{12} \mathrm{CO}(1-0)$ and ${ }^{13} \mathrm{CO}(1-0)$ velocity channel maps}

Figure 3 shows the velocity channel maps observed in ${ }^{12} \mathrm{CO}(1-0)$ and ${ }^{13} \mathrm{CO}(1-0)$. In the maps for $V_{\mathrm{LSR}}<3.7 \mathrm{~km} \mathrm{~s}^{-1}$, both ${ }^{12} \mathrm{CO}(1-0)$ and ${ }^{13} \mathrm{CO}(1-0)$ emission is seen in the northeastern part of the maps (RA, Dec $=4: 24: 00,28: 15: 00)$. In the channel maps for $4.0<V_{\mathrm{LSR}}<7.3 \mathrm{~km} \mathrm{~s}^{-1}$, enhanced emission is seen toward the B211/B213 filament in both ${ }^{12} \mathrm{CO}(1-0)$ and ${ }^{13} \mathrm{CO}(1-0)$. The emission at these velocities is likely to be directly associated with the B211/B213 filament. Furthermore, while the emission at $4 \mathrm{~km} \mathrm{~s}^{-1}<V_{\mathrm{LSR}}<6 \mathrm{~km} \mathrm{~s}^{-1}$ is distributed southwest of the B211/B213 filament, the emission at $6 \mathrm{~km} \mathrm{~s}^{-1}$ $<V_{\mathrm{LSR}}<7 \mathrm{~km} \mathrm{~s}^{-1}$ is distributed northeast of the filament. In the channel maps for $V_{\mathrm{LSR}}>7.3 \mathrm{~km} \mathrm{~s}^{-1}$, the distribution of the ${ }^{12} \mathrm{CO}(1-0)$ and ${ }^{13} \mathrm{CO}(1-0)$ emission is suggestive of an arc-like structure around L1495. Figure 1 (right) is a sketch showing the location of each velocity component.

\section{Modeling the data and discussion}

\subsection{D morphology of the B211/B213 ambient cloud}

Here, we discuss the 3D morphology of the material surrounding the B211/B213 filament by comparing the extent of the gas in the plane of the sky and its depth along the line of sight. Hereafter, we refer to the system consisting of the B211/B213 filament and its surrounding gas as the B211/B213 cloud (i.e., red, green, and dark blue areas in Fig. 1, right).

The projected extent of the B211/B213 cloud in the plane of the sky exceeds $\sim 10 \mathrm{pc}$. Taking the viewing angle into account, the real extent of the cloud may be larger. At the same time, we can estimate the depth of the cloud along the line of sight under the assumption that the surrounding material is filled by gas with a density exceeding the critical density of the ${ }^{13} \mathrm{CO}(1-0)$ line, since ${ }^{13} \mathrm{CO}(1-0)$ emission is observed throughout the entire mapped area. The critical density of ${ }^{13} \mathrm{CO}(1-0), n_{\text {critical }}$, may be estimated as follows:

$n_{\text {critical }}^{13}=\frac{A_{\mathrm{ul}}}{\sigma_{\text {cross }} v}=\frac{A_{\mathrm{ul}}}{10^{-15} \mathrm{~cm}^{-2} \times 10^{4} \sqrt{T_{\mathrm{ex}}}}$,

where $A_{\mathrm{ul}}, \sigma_{\text {cross }}, v$, and $T_{\text {ex }}$ are the Einstein spontaneous emission coefficient, collision cross section, collision velocity, and line excitation temperature. The values of $A_{10}$ and $\sigma_{\text {cross }}$ in the LAMDA database ${ }^{4}$ are $6.294 \times 10^{-8} \mathrm{~s}^{-1}$ and $10^{-15} \mathrm{~cm}^{-2}$. The collision velocity can be calculated as $v=\sqrt{3 k_{\mathrm{B}} T_{\mathrm{ex}} / m}=$ $10^{4} \sqrt{T_{\mathrm{ex}}} \mathrm{cm} \mathrm{s}^{-1}$, where $k_{\mathrm{B}}$ is the Boltzmann constant and $m$ is the hydrogen molecular mass. This leads to a value of $1.7 \times$ $10^{3} \mathrm{~cm}^{-3}$ for the critical density of ${ }^{13} \mathrm{CO}(1-0)$ assuming $T_{\mathrm{ex}} \simeq$ $14 \mathrm{~K}$. Here, we assumed that the excitation temperature $T_{\mathrm{ex}}$ of the ${ }^{13} \mathrm{CO}(1-0)$ line is the same as the dust temperature $T_{\text {dust }} \sim$ $14 \mathrm{~K}$ derived by Palmeirim et al. (2013) from Herschel data in the ambient cloud around B211/B213 (red and dark blue area in Fig. 1, right). Palmeirim et al. (2013) also estimated the mean Herschel column density in the material surrounding the B211/B213 filament to be $N_{\mathrm{H}_{2}} \simeq 1.4 \times 10^{21} \mathrm{~cm}^{-2}$. Thus, the depth of the cloud $\left(=\mathrm{N}_{\mathrm{H}_{2}} / n_{\text {critical }}{ }^{13} \mathrm{CO}\right.$ is estimated to be $0.3 \mathrm{pc}$.

Recently, Qian et al. (2015) independently estimated the depth of the whole Taurus molecular cloud and found a value of $\sim 0.7 \mathrm{pc}$ using the core velocity dispersion (CVD) method. With a projected extent of more than $10 \mathrm{pc}$ and a depth of $\sim 0.3-0.7 \mathrm{pc}$, we conclude that the 3D morphology of the cloud resembles a sheet-like structure (see Fig. 4). From $\mathrm{HC}_{3} \mathrm{~N}$ (2-1) and (10-9) observations, Li \& Goldsmith (2012) found that the depth of the dense $\left(\sim 10^{4} \mathrm{~cm}^{-3}\right)$ portion of the B213 region (i.e., the dense filament) is $\sim 0.12 \mathrm{pc}$. This is smaller than our estimate for the depth of the ambient molecular gas, but consistent with the view that the dense inner part of the B213 filament is a cylinder-like structure of $\sim 0.1 \mathrm{pc}$ diameter (Palmeirim et al. 2013), embedded in a lower-density sheet-like cloud.

\subsection{Accretion of background gas into the B211/B213 filament}

The blueshifted and redshifted emission components in both ${ }^{12} \mathrm{CO}(1-0)$ and ${ }^{13} \mathrm{CO}(1-0)$ are distributed southwest and northeast of the B211/B213 filaments (see Fig. 1 and Sect. 3.2). This morphology is suggestive of the mass accretion into the B211/B213 filament. In this suggestion, we compare the velocity pattern seen in ${ }^{12} \mathrm{CO}(1-0)$ and ${ }^{13} \mathrm{CO}(1-0)$ with the prediction of an accretion gas model, in order to investigate whether the B211/B213 filament accretes ambient cloud gas from a kinematic viewpoint.

\subsubsection{Observed position-velocity diagrams}

As mentioned in Sect. 3.2, the highly blueshifted and redshifted components at $V_{\mathrm{LSR}}<3.7 \mathrm{~km} \mathrm{~s}^{-1}$ and $V_{\mathrm{LSR}}>7.3 \mathrm{~km} \mathrm{~s}^{-1}$ do not seem to be directly connected to the B211/B213 cloud/filament.

4 http://home.strw.leidenuniv.nl/ moldata/datafiles/ 13 co.dat 

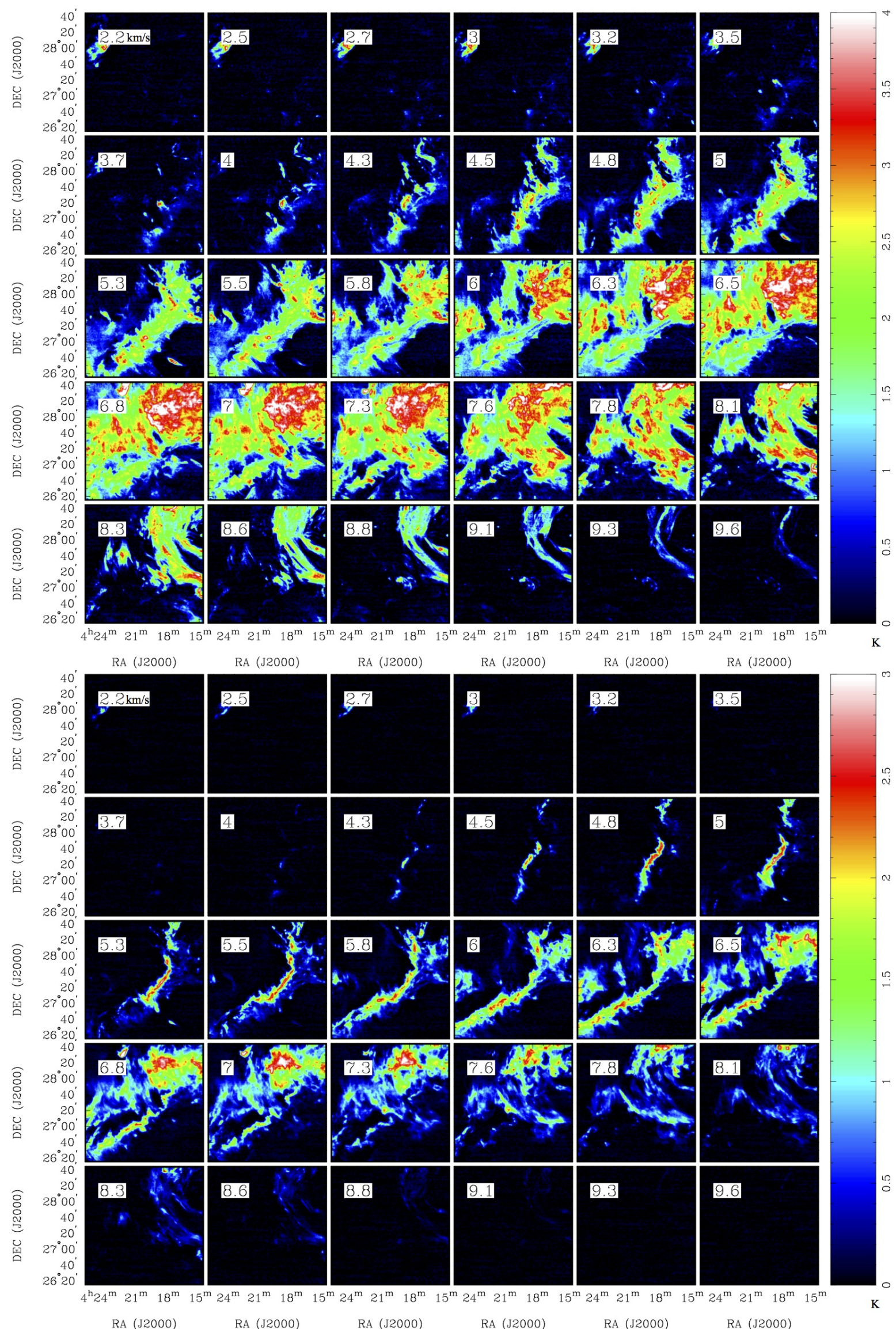

Fig. 3. Velocity channel maps in the ${ }^{12} \mathrm{CO}(J=1-0$, top panel $)$ and ${ }^{13} \mathrm{CO}(J=1-0$, bottom panel $)$ emission lines in units of $\mathrm{K}$ obtained from the Goldsmith et al. (2008) data. The line of the sight (LSR) velocities (in $\mathrm{km} \mathrm{s}^{-1}$ ) are indicated in the top left corner of each panel. The velocity width of each channel map is $0.3 \mathrm{~km} \mathrm{~s}^{-1}$. 

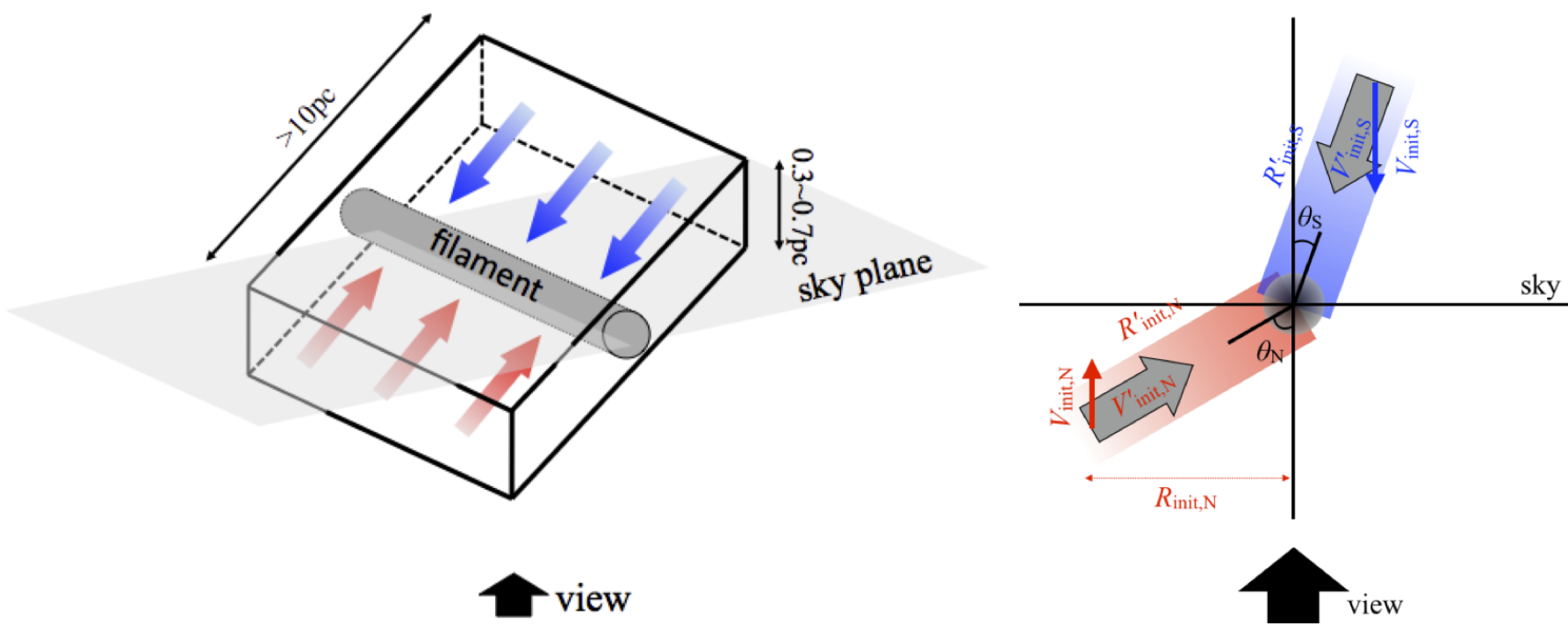

Fig. 4. Left panel: schematic picture of the structure of the B211/B213 cloud (see Sect. 4.1). Right panel: schematic picture of our toy model of the velocity field (see Sect. 4.2.2).
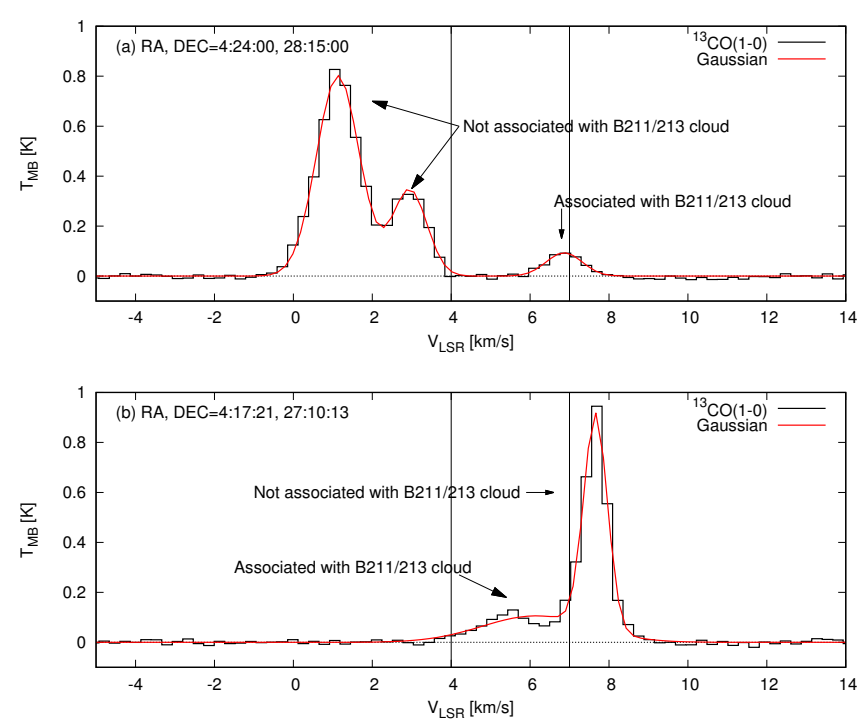

Fig. 5. Schematic picture of the definition of velocity components associated with the B211/B213 filament. The spectrum in each panel is the ${ }^{13} \mathrm{CO}(1-0)$ spectrum averaged over a $15^{\prime} \times 15^{\prime}$ area with a center position indicated in the top-left corner. The velocity components with a velocity of $<4.0 \mathrm{~km} \mathrm{~s}^{-1}$ or $>7.0 \mathrm{~km} \mathrm{~s}^{-1}$ are regarded as components not associated with the B211/B213 filament. These components are subtracted from the data cube.

To focus on the velocity field of the gas associated with the B211/B213 filament, we subtracted these two components as follows. We applied Gaussian fitting with $N$ Gaussian components to each pixel, where $N=1,2,3,4$, or 5 . When the $\mathrm{S} / \mathrm{N}$ of the residual peak intensity was lower than 5 , the fit was deemed to be acceptable and the corresponding spectrum was assumed to consist of $N$ Gaussian components. Then, if the peak LSR velocity of a Gaussian component was lower than $4.0 \mathrm{~km} \mathrm{~s}^{-1}$ or higher than $7.0 \mathrm{~km} \mathrm{~s}^{-1}$, the component was not considered to be associated with the B211/B213 filament or cloud and was subtracted from the data cube (see also Figs. 5 and A.1). Figure A.2 displays the ${ }^{12} \mathrm{CO}(1-0)$ and ${ }^{13} \mathrm{CO}(1-0)$ velocity channel maps after subtracting these components. Hereafter, we used these subtracted data cubes.
Figure 6 shows the resulting position-velocity $(P V)$ diagrams in ${ }^{12} \mathrm{CO}(1-0)$ and ${ }^{13} \mathrm{CO}(1-0)$ along a direction perpendicular to the B211/B213 filament as indicated in Fig. 1. In these $P V$ diagrams, distinct velocity pattern can be recognized in ${ }^{12} \mathrm{CO}$ $(1-0)$ and ${ }^{13} \mathrm{CO}(1-0)$ toward the filament (loffsetl $<10^{\prime} \sim 0.4 \mathrm{pc}$ ). This is probably due to differing optical depths in the two lines. As described in Sect. 3.1, the ${ }^{12} \mathrm{CO}(1-0)$ optical depth toward the filament is $>50$ and much larger than the optical depth toward the outskirts of the filament, suggesting that the ${ }^{12} \mathrm{CO}(1-0)$ emission only traces the surface of the filament. In the outskirts of the B211/B213 filament (loffsetl $>10^{\prime}$ ), the blueshifted emission is distributed southwest (offset $>0^{\prime}$ ) and the redshifted emission is distributed northeast (offset $<0^{\prime}$ ) of the filament. The velocities of the blueshifted and redshifted components approach the velocity of the B211/B213 filament as the offset approaches 0 (i.e., the crest of the filament). Transverse velocity gradients perpendicular to the major axis of filaments have also been observed toward several dense filaments in the Serpens cloud (Dhabal et al. 2018) as well as toward the main filament in the northwestern part of the L1495 subregion (Arzoumanian et al. 2018).

\subsubsection{Gas accretion model}

The $P V$ diagrams in Fig. 6 show an asymmetric velocity distribution on either side of the 0 position (filament crest), suggesting that the sheet-like ambient cloud surrounding the B211/B213 filament has a different inclination to the plane of the sky northeast and southwest of the filament. To investigate whether the B211/B213 filament accretes gas from the ambient cloud, we thus constructed a three-component toy model (one filament component and two components for the northeastern and southwestern sheets) under the assumption that the sheet components northeast (redshifted) and southwest (bluesshifted) lie on the near and far sides of the B211/B213 filament, respectively, as shown in Fig. 4. Our modeling procedure is summarized in the schematic picture shown in Fig. A.3.

Model for the central filament component. First, we produced a model for the filament. Herschel observations of nearby clouds have shown that the radial column density profiles of molecular filaments in the radial direction $R^{\prime}$ (i.e., perpendicular to the filament crest) can be well described by the following 

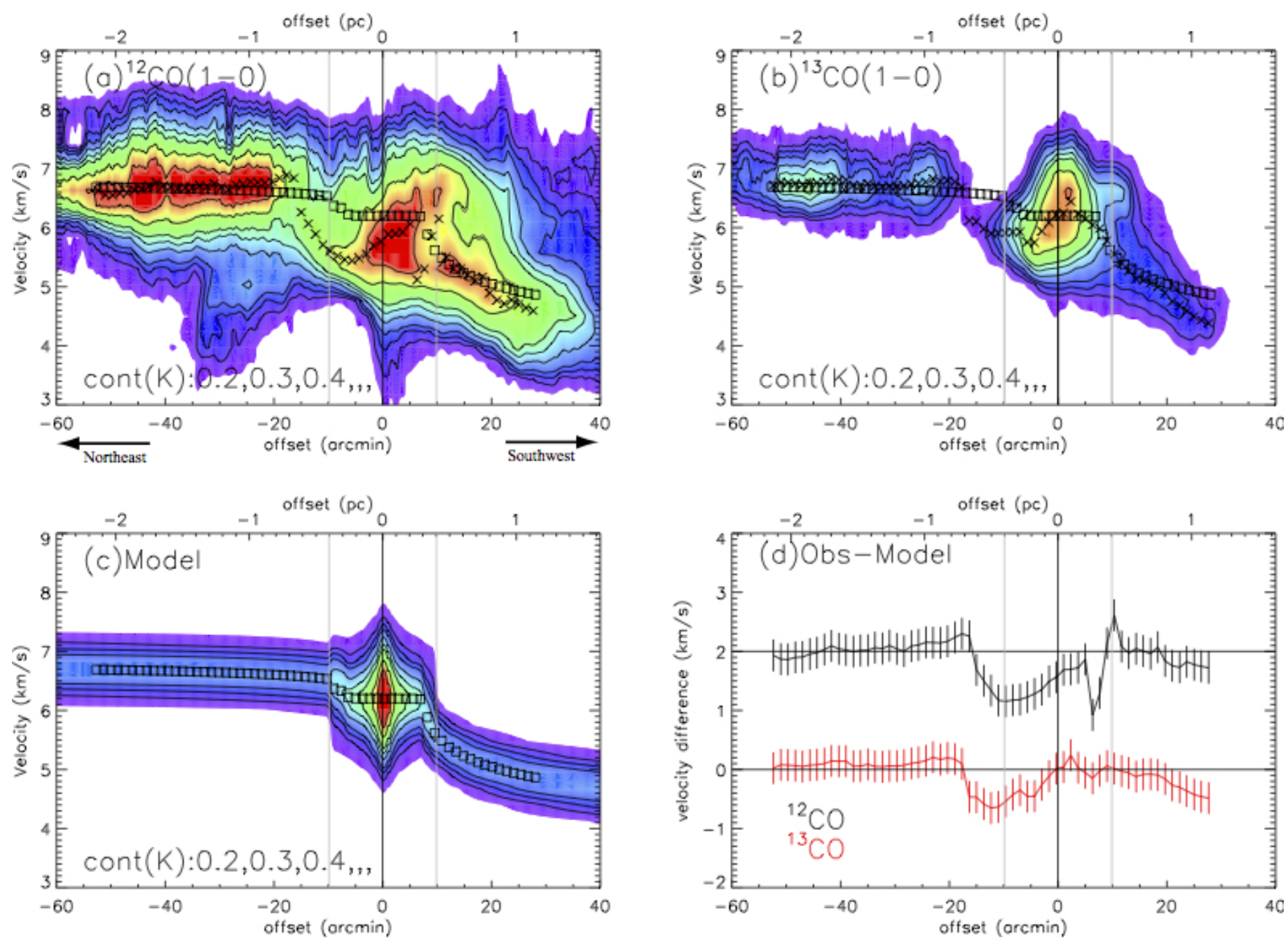

Fig. 6. Position-velocity diagram of ${ }^{12} \mathrm{CO}(1-0)$ (panel a), ${ }^{13} \mathrm{CO}(1-0)($ panel $b$ ), and the model (panel c) and velocity (panel $d$ ) offsets between ${ }^{12} \mathrm{CO}(1-0)$ and ${ }^{13} \mathrm{CO}(1-0)$ observations and model. The assumed parameters for the accretion model are summarized in Table 1 . The cut line of the $P V$ diagrams is indicated in Fig 1 . In panels $a-c$, black squares indicate the peak velocity positions at each offset. In panels $a$ and $b$, black crosses are the peak velocity positions at each offset in the model. In panel $d$, lines indicate the velocity offset (black) between ${ }^{12} \mathrm{CO}(1-0)$ and the model and (red) between ${ }^{13} \mathrm{CO}(1-0)$ and the model. In panels $a-d$, black and gray vertical lines indicate offset $=0^{\prime}$ and loffsetl $<R_{\text {out }}$.

"Plummer-like" function (Arzoumanian et al. 2011; Palmeirim et al. 2013):

$\Sigma_{p}\left(R^{\prime}\right) / \mu m_{\mathrm{H}}=\frac{N_{\mathrm{H}_{2}}^{0}}{\left[1+\left(R^{\prime} / R_{\mathrm{flat}}\right)^{2}\right]^{\frac{p-1}{2}}} \quad \rightarrow \rho_{p}\left(R^{\prime}\right)=\frac{\rho_{\mathrm{c}}}{\left[1+\left(R^{\prime} / R_{\mathrm{flat}}\right)^{2}\right]^{\frac{p}{2}}}$,

where $\rho_{\mathrm{c}}, \Sigma_{p}, \mu, m_{\mathrm{H}}, N_{\mathrm{H}_{2}}^{0}, p$, and $R_{\mathrm{flat}}$ are the central density of the filament, the column density as a function of radius $R^{\prime}$, the mean molecular mass, the hydrogen atom mass, the central column density, the index of the power-law density profile at large radii $\left(R^{\prime} \gg R_{\text {flat }}\right)$, and the radius of the flat inner region, respectively. For the B211/B213 filament, we adopted $N_{\mathrm{H}_{2}}^{0}=1.4 \times 10^{21} \mathrm{~cm}^{-2}$, $p=2.0$, and $R_{\text {flat }}=0.03$ pc from the fitting results of Palmeirim et al. (2013). We assumed that the filament itself lies in the plane of the sky and that the shape of the intensity profile of the B211/B213 filament as traced in ${ }^{12} \mathrm{CO}(1-0)$ and ${ }^{13} \mathrm{CO}(1-0)$ emission is the same as that found in the Herschel column density map. Then, we rescaled the peak integrated intensity to be $2 \mathrm{~K} \mathrm{~km} \mathrm{~s}^{-1}$ as observed in ${ }^{13} \mathrm{CO}(1-0)$.

Approximating the Plummer density profile of the filament by a broken power-law, the gravitational potential in the radial direction $R^{\prime}$ can be expressed as follows ${ }^{5}$ (cf. Hennebelle \& André 2013):

$\phi\left(R^{\prime}\right)= \begin{cases}G \rho_{\text {flat }} \pi R^{\prime 2}, & \text { for } R^{\prime} \leq R_{\text {flat }}, \\ G M_{\text {line,flat }}\left[1+2 \ln \left(\frac{R^{\prime}}{R_{\text {flat }}}\right)+2\left(\ln \frac{R^{\prime}}{R_{\text {flat }}}\right)^{2}\right], & \text { for } R_{\text {flat }}<R^{\prime} \leq R_{\text {out }}, \\ G M_{\text {line,flat }}\left[1+2 \ln \left(\frac{R_{\text {out }}}{R_{\text {flat }}}\right)+2\left(\ln \frac{R_{\text {out }}}{R_{\text {flat }}}\right)^{2}\right], & \\ +2 G M_{\text {line }} \ln \left(\frac{R^{\prime}}{R_{\text {out }}}\right), & \text { for } R_{\text {out }}<R^{\prime},\end{cases}$

where $\rho_{\text {flat }}$ and $R_{\text {out }}$ are the density of the filament at $R^{\prime} \leq$ $R_{\text {flat }}$ and outer radius of the filament, respectively. We adopted $n_{\mathrm{H}_{2}}^{0}=\rho_{\text {flat }} / \mu m_{\mathrm{H}}=4.5 \times 10^{4} \mathrm{~cm}^{-3}, R_{\text {flat }}=0.03 \mathrm{pc}$, and $R_{\text {out }}=$ 0.4 pc from Palmeirim et al. (2013), as summarized in Table 1. In the above equation, $M_{\text {line,flat }}$ and $M_{\text {line }}$ represent the inner and

5 Here, $R^{\prime}$ denotes the radius corrected for inclination to the line of sight, where the relation between the corrected radius $R^{\prime}$ and radius in the sky plane $R$ is $R^{\prime}=R / \sin \left(\theta_{\mathrm{N} / \mathrm{S}}\right)$ and $\theta_{\mathrm{N} / \mathrm{S}}$ is the inclination angle of the northeastern and southwestern sheet component to the line of sight. 
Table 1. Properties of the three modeled cloud components.

\begin{tabular}{ccc}
\hline \hline Component & Parameter & \\
\hline & $M_{\text {line }}$ & $54 M_{\odot} \mathrm{pc}^{-1 b}$ \\
Filament $^{a}$ & $n_{\mathrm{H}_{2}}$ & $4.5 \times 10^{4} \mathrm{~cm}^{-3 b}$ \\
& $p$ & $2^{b}$ \\
& $R_{\text {flat }}$ & $0.03 \mathrm{pc}^{b}$ \\
& $R_{\text {out }}$ & $0.4 \mathrm{pc}^{b}$ \\
& $\mathcal{V}_{\text {filament }}$ & $6.2 \mathrm{~km} \mathrm{~s}^{-1 c}$ \\
\hline & $\mathcal{V}_{\text {init,N }}$ & $6.8 \mathrm{~km} \mathrm{~s}^{-1 c}$ \\
& $R_{\text {init, }}^{\prime}$ & $10 \mathrm{pc}^{\prime}$ \\
& $\theta_{\mathrm{N}}$ & $70 \mathrm{deg}$ \\
\hline \multirow{3}{*}{ Sortheastern sheet } & \\
& $\mathcal{V}_{\text {init,S }}$ & $4.4 \mathrm{~km} \mathrm{~s}$ s $^{-1 c}$ \\
& $R_{\text {init,S }}^{\prime}$ & $10 \mathrm{pc}$ \\
& $\theta_{\mathrm{S}}$ & $20 \mathrm{deg}$ \\
\hline
\end{tabular}

Notes. ${ }^{(a)}$ The total masses of the filament, northeastern sheet, and southwestern sheet components are estimated to be $\sim 400, \sim 700$, and $\sim 300 M_{\odot}$, respectively, from the Herschel $\mathrm{H}_{2}$ column density map. ${ }^{(b)}$ Adopted from Palmeirim et al. (2013). ${ }^{(c)}$ Peak velocity at $R_{\text {init }}$.

total masses per unit length of the filament and are given by

$$
\begin{aligned}
& M_{\text {line,flat }}=\rho_{\text {flat }} \pi R_{\text {flat }}^{2}, \\
& M_{\text {line }}=\rho_{\text {flat }} \pi R_{\text {flat }}^{2}\left[1+2 \ln \left(\frac{R_{\text {out }}}{R_{\text {flat }}}\right)\right] .
\end{aligned}
$$

Models for the northeastern and southwestern sheet components. Second, we produced models for the northeastern and southwestern sheet components assuming that the B211/B213 filament accretes the gas of the sheets as a result of its gravitational potential.

Taking into account the pressure gradient force, conservation of energy for a parcel of unit mass of the ambient cloud falling onto the central filament may be expressed as follows (cf. Smith 1994, 2012):

$$
\begin{aligned}
\frac{1}{2}\left(\mathcal{V}_{\mathrm{init}, \mathrm{N} / \mathrm{S}}^{\prime 0}\right)^{2}+ & \phi\left(R_{\mathrm{init}, \mathrm{N} / \mathrm{S}}^{\prime}\right)+C_{\mathrm{s}, \mathrm{eff}}^{2} \ln \left(\rho_{\mathrm{init}}\right) \\
& =\frac{1}{2} \mathcal{V}\left(R^{\prime}\right)^{2}+\phi\left(R^{\prime}\right)+C_{\mathrm{s}, \mathrm{eff}}^{2} \ln \left(\rho\left(R^{\prime}\right)\right)
\end{aligned}
$$

where $\mathcal{V}(R)$ is the projected velocity and $C_{\mathrm{s}, \text { eff }}$ is the effective sound speed. The projected velocity $\mathcal{V}(R)$ of the gas flow can thus be expressed as follows:

$$
\begin{aligned}
& \mathcal{V}(R)=\mathcal{V}_{\text {filament }} \\
& \pm \sqrt{2\left[\frac{1}{2}\left(\mathcal{V}_{\text {init,N/S}}^{\prime 0}\right)^{2}+\phi\left(R_{\text {init,N/S}}^{\prime}\right)-\phi\left(R^{\prime}\right)+C_{\mathrm{s}, \text { eff }}^{2} \ln \left(\frac{\rho_{\text {init }}}{\rho\left(R^{\prime}\right)}\right)\right]} \\
& \times \cos \left(\theta_{\mathrm{N} / \mathrm{S}}\right),
\end{aligned}
$$

where $\mathcal{V}_{\text {filament }}, \mathcal{V}_{\text {init,N/S}}^{\prime 0}, R_{\text {init,N/S }}^{\prime}$, and $\rho_{\text {init }}$ are the systemic velocity of the filament, the velocity of the accreting gas at the initial point corrected for inclination, the initial radius of the accreting gas corrected for inclination, and the volume density at the initial point, respectively. Here, we define $\mathcal{V}_{\text {init,N/S }}^{\prime 0}$ as $\left(\mathcal{V}_{\text {init,N/S }}-\mathcal{V}_{\text {filament }}\right) / \cos \left(\theta_{\mathrm{N} / \mathrm{S}}\right)$, where $\mathcal{V}_{\text {init,N/S }}$ is the projected velocity of the northeastern and southwestern sheet component at $R_{\text {init,N/S }}^{\prime}$. We adopted $C_{\mathrm{s}, \text { eff }}=0.9 \mathrm{~km} \mathrm{~s}^{-1}$ from

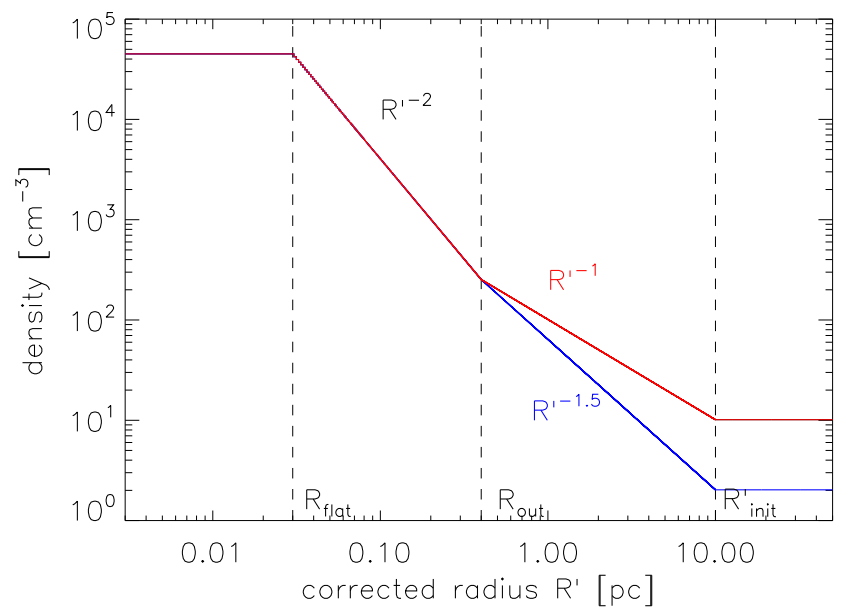

Fig. 7. Assumed density profile for the three-component model. Red and blue lines indicate the densities for the northeastern and southwestern sheet components, respectively.

$C_{\mathrm{s}, \mathrm{eff}} \simeq \delta V_{\mathrm{FWHM}}\left({ }^{12} \mathrm{CO}\right) / \sqrt{8 \ln 2}$, where $\delta V_{\mathrm{FWHM}}\left({ }^{12} \mathrm{CO}\right)$ is the ${ }^{12} \mathrm{CO}(1-0)$ line width $\left(=2.1 \mathrm{~km} \mathrm{~s}^{-1}\right)$ observed toward the B211/B213 filament. When the value of $C_{\mathrm{s}, \text { eff }}^{2} \ln \left(\frac{\rho_{\text {init }}}{\rho\left(R^{\prime}\right)}\right)$ was higher than $\frac{1}{2}\left(\mathcal{V}_{\text {init,N/S}}^{\prime 0}\right)^{2}+\phi\left(R_{\text {init, } / \mathrm{S}}^{\prime}\right)-\phi\left(R^{\prime}\right)$, we adopted $\mathcal{V}(R)=$ $\mathcal{V}_{\text {filament }}$. The Herschel observations show that the density profile of the B211/B213 filament is proportional to $R^{\prime-2}$ at $R^{\prime} \leq R_{\text {out }}^{\prime}$ and has a shallower slope at $R^{\prime} \geq R_{\text {out }}^{\prime}$ (Palmeirim et al. 2013). Furthermore, the slope for the southwestern sheet component is slightly steeper than the slope for the northeastern sheet component. At $R^{\prime}>R_{\text {init }}^{\prime}$, the gas density in the model was assumed to be constant.

To summarize, we assumed the following density distribution as a function of radial direction $R^{\prime}$ (see Fig. 7):

For the northeastern sheet component,

$$
\begin{aligned}
& \rho\left(R^{\prime}\right)= \\
& \begin{cases}\rho_{\text {flat }}, & \text { for } R^{\prime} \leq R_{\text {flat }}, \\
\rho_{\text {flat }}\left(\frac{R^{\prime}}{R_{\text {flat }}}\right)^{-2}, & \text { for } R_{\text {flat }}<R^{\prime} \leq R_{\text {out }}^{\prime}, \\
\rho_{\text {flat }}\left(\frac{R_{\text {out }}}{R_{\text {flat }}}\right)^{-2}\left(\frac{R^{\prime}}{R_{\text {out }}}\right)^{-1.0}, & \text { for } R_{\text {out }}^{\prime}<R^{\prime} \leq R_{\text {init }}^{\prime}, \\
\text { constant }=\rho_{\text {flat }}\left(\frac{R_{\text {out }}}{R_{\text {flat }}}\right)^{-2}\left(\frac{R_{\text {init }}^{\prime}}{R_{\text {out }}}\right)^{-1.0}, & \text { for } R_{\text {init }}^{\prime}<R^{\prime}\end{cases}
\end{aligned}
$$

For the southwestern sheet component,

$$
\begin{aligned}
& \rho\left(R^{\prime}\right)= \\
& \begin{cases}\rho_{\text {flat }}, & \text { for } R^{\prime} \leq R_{\text {flat }}, \\
\rho_{\text {flat }}\left(\frac{R^{\prime}}{R_{\text {flat }}}\right)^{-2}, & \text { for } R_{\text {flat }}<R^{\prime} \leq R_{\text {out }}^{\prime}, \\
\rho_{\text {flat }}\left(\frac{R_{\text {out }}}{R_{\text {flat }}}\right)^{-2}\left(\frac{R^{\prime}}{R_{\text {out }}}\right)^{-1.5}, & \text { for } R_{\text {out }}^{\prime}<R^{\prime} \leq R_{\text {init }}^{\prime}, \\
\text { constant }=\rho_{\text {flat }}\left(\frac{R_{\text {out }}}{R_{\text {flat }}}\right)^{-2}\left(\frac{R_{\text {init }}^{\prime}}{R_{\text {out }}}\right)^{-1.5}, & \text { for } R_{\text {init }}^{\prime}<R^{\prime} .\end{cases}
\end{aligned}
$$

We also assumed that both sheet components have integrated intensities of $\sim 1 \mathrm{~K} \mathrm{~km} \mathrm{~s}^{-1}$ as observed in ${ }^{13} \mathrm{CO}(1-0)$. To obtain a good agreement between the models and the observations (see Appendix B), we adopted $\theta_{\mathrm{N}}=70^{\circ}, \mathcal{V}_{\text {init,N }}=6.8 \mathrm{~km} \mathrm{~s}^{-1}$, and $R_{\text {init,N }}^{\prime}=10 \mathrm{pc}$ for the northeastern sheet component and $\theta_{\mathrm{S}}=$ $20^{\circ}, \mathcal{V}_{\text {init,S }}=4.4 \mathrm{~km} \mathrm{~s}^{-1}$, and $R_{\text {init,S }}^{\prime}=10 \mathrm{pc}$ for the southwestern sheet component. The parameters of our model are summarized in Table 1. For simplicity, we assumed constant inclinations to the line of sight of $70^{\circ}$ for the northeastern sheet component and 
$20^{\circ}$ for the southwestern sheet component in our model. In reality, however, the inclinations of the two components may vary smoothly with radius from the B211/B213 filament and match on the filament crest.

Combined three-component model. We first generated an integrated intensity distribution and a peak velocity field for each of the three components with IDL (Interactive Data Language). Using the MIRIAD task velimage ${ }^{6}$, we then produced individual data cube components for the filament and the two sheet components assuming uniform velocity dispersions of $1.3 \mathrm{~km} \mathrm{~s}^{-1}$ for the filament and $0.9 \mathrm{~km} \mathrm{~s}^{-1}$ for the sheet components. The velocity dispersions were obtained from fitting the observed ${ }^{13} \mathrm{CO}(1-0)$ spectra. One of the reasons for the larger velocity dispersion observed in ${ }^{13} \mathrm{CO}$ toward the central filament may be that the B211/B213 filament contains several velocity subcomponents (or "fiber-like" structures; Hacar et al. 2013), possibly as a result of accretion-driven turbulence (cf. Hennebelle \& André 2013; Heitsch 2013; André et al. 2014) ${ }^{7}$. Finally, we used IDL to coadd the three individual data-cube components and produce a combined model data cube.

Large-scale kinematic model. We adopted initial velocities (corrected for inclination) of $\mathcal{V}_{\text {init, } \mathrm{N}}^{\prime 0}=1.8 \mathrm{~km} \mathrm{~s}^{-1}\left(=\left[\mathcal{V}_{\text {init,N }}-\right.\right.$ $\left.\left.\mathcal{V}_{\text {filament }}\right] / \cos \left(\theta_{\mathrm{N}}\right)\right)$ for the northeastern sheet component and $\mathcal{V}_{\text {init,S }}^{\prime 0}=-1.9 \mathrm{~km} \mathrm{~s}^{-1}\left(=\left[\mathcal{V}_{\text {init,S }}-\mathcal{V}_{\text {filament }}\right] / \cos \left(\theta_{\mathrm{S}}\right)\right)$ for the southwestern sheet component. This almost symmetric initial velocity pattern after correction for inclination is suggestive of gravitational accretion. If the accreting gas comes from far away positions $R_{\text {far, } / \mathrm{S}}^{\prime}\left(\gg R_{\text {init }}^{\prime}\right)$ and is accelerated by the gravitational potential of the B211/B213 filament/cloud, the lineof-sight velocity at $R_{\text {far, } / \mathrm{S}}^{\prime}$ is likely to be similar to $\mathcal{V}_{\text {filament. The }}$ positions $R_{\text {far, N/S }}^{\prime}$ for the northeastern and southwestern sheet components can be estimated from the equation of $\mathcal{V}_{\text {init, } / \mathrm{S}}^{\prime}=$ $\sqrt{2\left[\phi\left(R_{\text {far }, \mathrm{N} / \mathrm{S}}^{\prime}\right)-\phi\left(R_{\text {init,N/S}}^{\prime}\right)\right]} \times \cos \left(\theta_{\mathrm{N} / \mathrm{S}}\right)$ since the pressure density gradient is probably small and can be neglected. We adopted $R_{\text {init, }}^{\prime}=10 \mathrm{pc}$ and $R_{\text {init, }}^{\prime}=10 \mathrm{pc}$. Thus, assuming that the initial velocities are entirely generated by gravitational acceleration, the surrounding gas for the northeastern and southwestern sheet components would have to come from $R_{\text {far, }}^{\prime}\left(R_{\text {far,N }}\right)=$ 270 (260) pc and $R_{\text {far }, \mathrm{S}}^{\prime}\left(R_{\mathrm{far}, \mathrm{S}}\right)=520$ (180) pc (see Fig. 8). Here, for simplicity, we did not include the mass of the sheets when we estimated the gravitational potential. Thus, these $R_{\mathrm{far}, \mathrm{N} / \mathrm{S}}^{\prime}$ values should be considered upper limits. The HI emission observed at $V_{\mathrm{LSR}} \sim 6 \mathrm{~km} \mathrm{~s}^{-1}$, which corresponds to the systemic velocity of the B211/B213 filament, has an extended emission with an extent of several $\times 100 \mathrm{pc}$, which is consistent with the above value of $R_{\text {far, N }}^{\prime}$ (see also Sect. 4.3 and Fig. A.4). Thus, one of the reasons why the initial velocities at $R_{\text {init, } / \mathrm{S}}^{\prime}$ in the northeastern and southwestern sheet components differ from the velocity of the filament may be the large-scale effect

\footnotetext{
6 velimage makes a data cube output_cube $\left(x, y, v_{\text {centroid }}\right)$ from an input-integrated intensity image input_intensity $(x, y)$, input-centroid velocity image input_velocity $(x, y)$, and dispersion $\sigma$. The $v_{\text {centroid }}-$ values are the centroid velocity and are input as an $(x, y)$ image. The output cube image is produced as output_cube $\left(x, y, v_{\text {centroid }}\right)=$ input_intensity $\left.(x, y) \times \exp \left(-\left(v_{\text {centroid }}-\text { input_velocity }(x, y)\right)^{2} /\left(2 \sigma^{2}\right)\right)\right)$.

7 In recent numerical simulations of this process, Seifried \& Walch (2015) did find that the accretion flow increases the velocity dispersion of the central filament, and Clarke et al. (2017) suggested that fiber-like structures could be produced as a result of the vorticity generated by an inhomogeneous accretion flow.
}

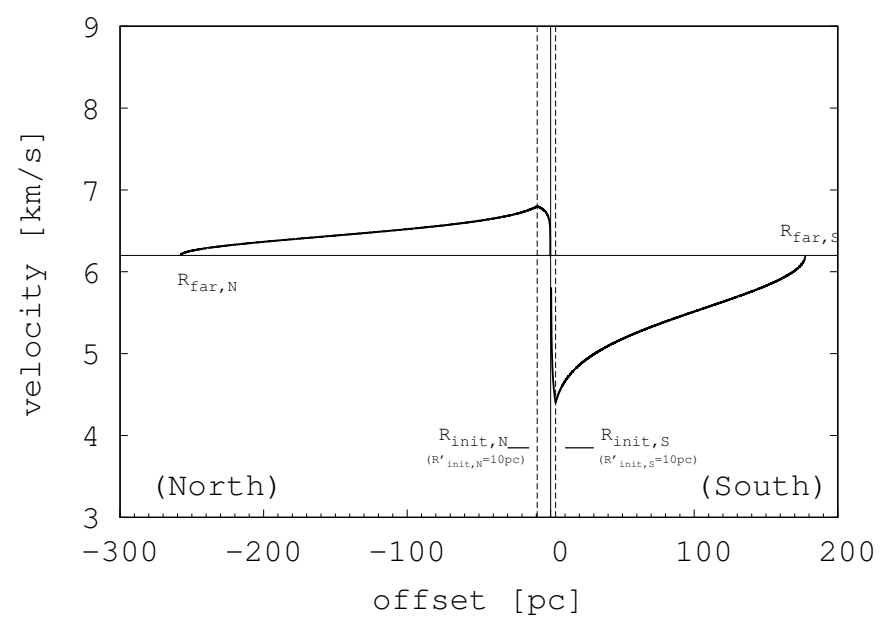

Fig. 8. Position-velocity diagram of the model for the large scale. $\theta_{\mathrm{N}}=70^{\circ}$ and $\theta_{\mathrm{S}}=20^{\circ}$ are assumed. Figure 6 corresponds to $-2.4 \mathrm{pc}<$ offset $<1.6 \mathrm{pc}$ in this figure. The vertical dashed lines mark $R_{\text {init,N }}=$ $-9.4 \mathrm{pc}\left(R_{\mathrm{init}, \mathrm{N}}^{\prime}=10 \mathrm{pc}\right)$ and $R_{\mathrm{init}, \mathrm{S}}=3.4 \mathrm{pc}\left(R_{\mathrm{init}, \mathrm{S}}^{\prime}=10 \mathrm{pc}\right)$.

of the gravitational potential of the B211/B213 cloud/filament. We discuss another possible explanation in Sect. 4.3.

\subsubsection{Comparing the combined model with the observations}

The synthetic $P V$ diagram predicted by the model is shown in Fig. 6c for comparison with the $P V$ diagrams observed in ${ }^{12} \mathrm{CO}(1-0)$ and ${ }^{13} \mathrm{CO}(1-0)$ (Figs. 6a and b). A good quantitative agreement especially with the ${ }^{13} \mathrm{CO}(1-0)$ diagram can be seen. In particular, in both the model and the observed $P V$ diagrams, the velocity of the gas surrounding the B211/B213 filament (red and dark blue area in Fig. 1, right) approaches the systemic velocity of the B211/B213 filament as the positional offset approaches 0 (i.e., the filament crest). While the gas is accelerated by the gravitational potential of the filament/cloud at large scales (several $\times 10 \mathrm{pc}$ ), it is decelerated by the pressure gradient force of the dense filament at small scales (several pc; see Figs. 8 and 9). The good agreement between the model and the data indicates that observational kinematic constraints are consistent with the B211/B213 filament accreting background cloud material as a result of its gravitational potential. This provides strong support to the scenario of mass accretion along magnetic field lines into the filament proposed by Palmeirim et al. (2013). The mass accretion rate onto the B211/B213 filament was estimated to be $27-50 M_{\odot} \mathrm{pc}^{-1} \mathrm{Myr}^{-1}$ by Palmeirim et al. (2013), suggesting that it took $\sim 1-2$ Myr to form the filament. Thus, accretion of gas from the ambient cloud in B211/B213 likely plays a key role in the evolution of the filament.

\subsection{Formation of the B211/B213 filament by large-scale compression?}

As described in Sect. 4.2, we adopted different inclinations for the northeastern sheet component $\left(i=70^{\circ}\right)$ and for the southwestern sheet component $\left(i=20^{\circ}\right)$ in our model to obtain a good agreement with the observations. This suggests that the B211/B213 cloud is shaped like a shell (see Fig. 9). One possibility is that this shell-like structure was produced by large-scale compression.

In this section, we investigate whether the cloud surrounding the B211/B213 filament is affected by large-scale flow phenomena using wide-field $\mathrm{H} \alpha$ maps that trace gas that 


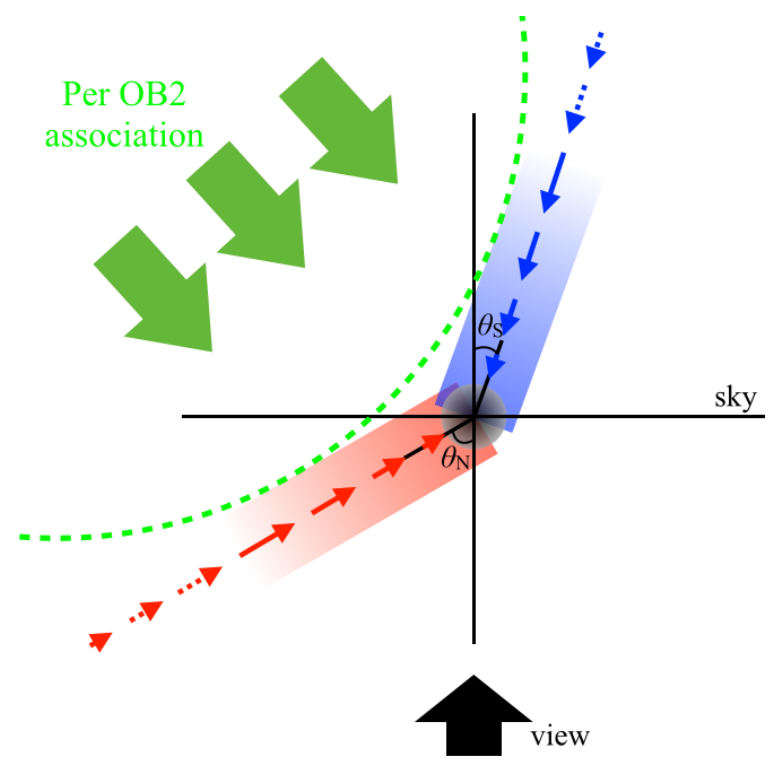

Fig. 9. Schematic picture of the relation between the B211/B213 cloud and Per OB2 association. The black arrows indicate the line of sight. The horizontal line indicate the sky plane. Red and blue arrows indicate the direction of the gas accretion in the northeastern and southwestern sheet components, respectively. Green arrows indicate the direction of the compression by Per OB 2 association. $\theta_{\mathrm{N}}$ and $\theta_{\mathrm{S}}$ are the inclination angles of the northeastern and southwestern sheet components to the line of sight. Red and blue arrows of length scaling quantitatively with the magnitude velocity field indicate the direction of the acceleration flow of ambient cloud material.

is ionized by massive stars (Finkbeiner 2003), the Planck $857 \mathrm{GHz}$ dust continuum map, which traces cold dust (Planck Collaboration I 2014), and an HI map that traces lower density atomic gas (HI4PI Collaboration 2016).

Figure 10 (see also Figs. A.4 and A.5) compare the spatial distributions of the $\mathrm{H} \alpha$ and $857 \mathrm{GHz}$ emission in the TaurusCalifornia-Perseus region (e.g., Taurus, Auriga, California, and Perseus). The $857 \mathrm{GHz}$ dust emission traces each molecular cloud and exhibits a hole-like structure. This hole-like structure can also be seen in HI emission, as shown in Figs. A.4 and A.5. The $\mathrm{H} \alpha$ emission fills the hole-like structure seen in the $857 \mathrm{GHz}$ dust emission near the center of the field. The Taurus, California, and Perseus molecular complexes traced by the $857 \mathrm{GHz}$ dust emission are distributed at the edge of the hole-like structure. Lim et al. (2013) also found evidence of a shell-like structure using dust extinction and ${ }^{12} \mathrm{CO}(1-0)$ maps. The hole-like structure may result from the expansion of a large-scale supershell produced by a supernova in the Per OB2 association that compresses the Taurus cloud from the far side (Olano \& Poeppel 1987; Bally et al. 2008). An $\mathrm{H} \alpha$ absorption feature is detected toward the Taurus cloud (see Figs. 10 and A.6), suggesting that the Taurus cloud lies at the front surface of the large-scale supershell produced by the Per OB2 association. The distance to the Per OB2 association is estimated to be $340 \mathrm{pc}$ from the Sun (Cernis 1993), while the distance to the Taurus cloud is $\sim 140 \mathrm{pc}$ (Elias 1978). These distances are consistent with the Taurus cloud lying in front of the Per OB2 association. The B211/B213 filament also appears to be in front of the HI shell (see Fig. 10 in Chapman et al. 2011). This morphology suggests that the B211/B213 filament may have formed as a result of an expanding supershell. This may provide another reason for the different initial gas velocities for the northeastern and southwestern sheet components in addition to large-scale

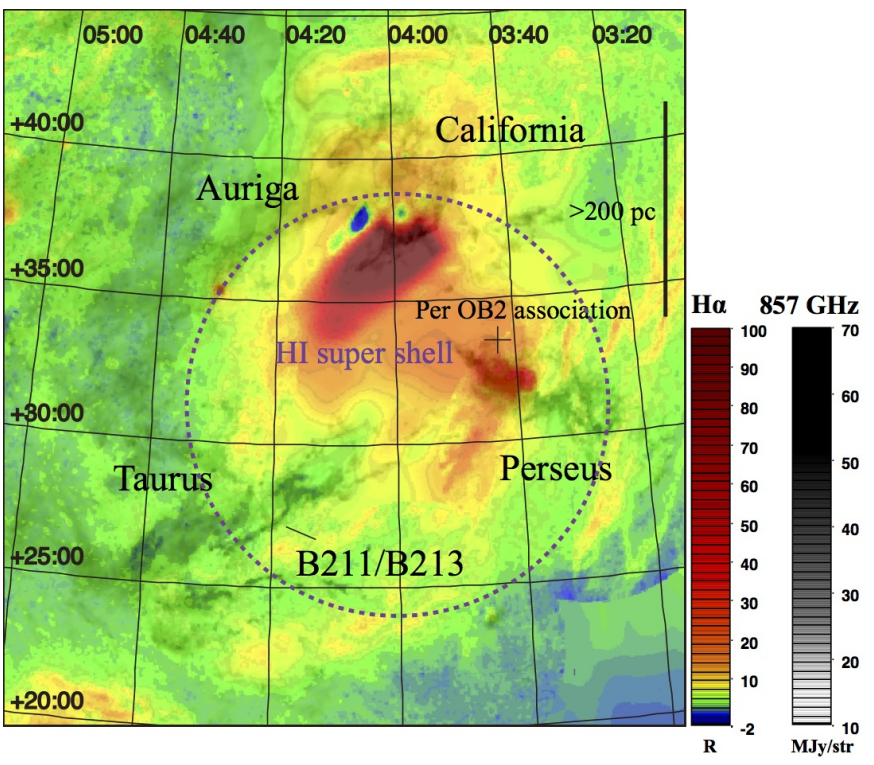

Fig. 10. Distributions of the $\mathrm{H} \alpha$ (color; Finkbeiner 2003) and $857 \mathrm{GHz}$ dust (gray; Planck Collaboration I 2014) emission. The units of the $\mathrm{H} \alpha$ and $857 \mathrm{GHz}$ maps are R (Rayleigh, $4 \pi \times 10^{-4}$ photons $\mathrm{cm}^{-2} \mathrm{~s}^{-1} \mathrm{sr}^{-1}$ ) and $\mathrm{MJy} \mathrm{str}^{-1}$, respectively. The magenta dashed circle indicates a HI supershell (Lim et al. 2013). The diameter of the HI supershell might be $>200 \mathrm{pc}$ since the distances to the Taurus and Perseus clouds are 140 and $340 \mathrm{pc}$, respectively. The distribution of HI emission is shown in Figs. A.4 and A.5. See also Fig. A.6.

acceleration by the gravitational potential of the B211/B213 cloud (see Sect. 4.2.3). The Local Bubble surrounding the Sun might also compress the Taurus cloud from the opposite direction. The Local Bubble surrounding the Sun was produced by supernovae (Snowden et al. 1998; Sfeir et al. 1999), and the wall of the Local Bubble is located close to the Taurus cloud (Könyves et al. 2007; Lallement et al. 2014).

Interestingly, the Planck $353 \mathrm{GHz}$ data show variations in the polarization fraction (i.e., polarized intensity/total intensity) across the B211/B213 filament, with lower and higher polarization fractions in the southwestern and northeastern parts of the filament, respectively (see Fig. 10 in Planck Collaboration Int. XXXIII 2016). If the gas surrounding the filament is shaped as a shell-like structure with an ordered magnetic field in the plane of each sheet component and if the southwestern sheet component is oriented closer to the line of the sight than the northeastern sheet component (cf. Fig. 4, right), the polarization fraction is expected to be lower in the southwestern area (dark blue in Fig. 1, right) than in the northeastern area (red in Fig. 1, right) assuming uniform dust grain properties. Moreover, both the polarization fraction and the polarization angle show smooth variations across the filament, which is consistent with the northeastern and southwestern sheets being curved (i.e., shell-like). The Planck polarization results therefore support the present model.

Using magnetohydrodynamic (MHD) numerical simulations, Inutsuka et al. (2015) and Inoue et al. (2018) have argued that multiple compressions associated with expanding bubbles can create star-forming filamentary structures within sheet-like molecular cloud. A similar model of anisotropic filament formation in shock-compressed layers has been proposed by Chen \& Ostriker (2014), also based on MHD simulations. Such anisotropic filament formation models naturally account for the transverse velocity gradients observed across the B211/B213 filament (see Fig. 1) and other dense molecular filaments 
(Dhabal et al. 2018), and agree well with the observational picture presented here. Another advantage of an MHD compression scenario is that it may naturally produce magnetically striations (Chen et al. 2017), as observed in the B211/B213 ambient cloud (see Sect. 1)

Based on these considerations, we propose the following scenario for the formation and evolution of the B211/B213 filamentary system:

1. A large-scale flow associated with the Per OB2 supershell compressed and deformed the cloud centered on the B211/B213 filament and created a bent shell-like structure.

2. Owing to its strong gravitational potential, the B211/B213 filament is growing in mass through accretion of background gas from the surrounding shell-like structure.

\section{Conclusions}

To examine whether the B211/B213 filament is accreting gas from the surrounding cloud, we investigated the velocity patterns observed in the ${ }^{12} \mathrm{CO}(1-0)$ and ${ }^{13} \mathrm{CO}(1-0)$ lines. Our main findings may be summarized as follows:

1. The optical depth of the ${ }^{12} \mathrm{CO}(1-0)$ line was estimated to be $\sim 3-300$. The ${ }^{12} \mathrm{CO}$ optical depth toward the B211/B213 filament is much larger than that toward the outskirts of the filament. The PV diagrams observed in ${ }^{12} \mathrm{CO}(1-0)$ and ${ }^{13} \mathrm{CO}(1-0)$ exhibit different velocity patterns close to the filament, which is likely due to different optical depths.

2. The ${ }^{12} \mathrm{CO}(1-0)$ and ${ }^{13} \mathrm{CO}(1-0)$ emission from the B211/B213 filament are seen at an LSR velocity of $\sim 6 \mathrm{~km} \mathrm{~s}^{-1}$. In the northeastern and southwestern parts of the $\mathrm{B} 211 / \mathrm{B} 213$ filament, the ${ }^{12} \mathrm{CO}(1-0)$ and ${ }^{13} \mathrm{CO}(1-0)$ emission is redshifted and blueshifted, respectively. The line-of-sight velocities gradually approach the systematic velocity of the filament closer to the filament.

3. The linear extent of the cloud around the B211/B213 filament is more than $10 \mathrm{pc}$ in the plane of the sky. In contrast, the depth of the cloud along the line of sight is estimated to be $\sim 0.3-0.7 \mathrm{pc}\left(=N_{\mathrm{H}_{2}} / n_{\text {critical }}\right)$ under the assumption that the density of the surrounding material is the same as the critical density of ${ }^{13} \mathrm{CO}(1-0)$. These results suggest that the 3D morphology of the gas cloud surrounding the B211/B213 filament is sheet-like.

4. To investigate whether the B211/B213 filament is in the process of accreting the surrounding gas material, we compared the velocity patterns observed in ${ }^{12} \mathrm{CO}(1-0)$ and ${ }^{13} \mathrm{CO}(1-0)$ with our three-component model. The predictions of the model were found to be in good agreement with the distribution of ${ }^{12} \mathrm{CO}(1-0)$ and ${ }^{13} \mathrm{CO}(1-0)$ emission in the observed PV diagrams, supporting the scenario of mass accretion along magnetic field lines into the B211/B213 filament proposed by Palmeirim et al. (2013).

5. From an inspection of the wide-field spatial distributions of $\mathrm{H} \alpha$ and $857 \mathrm{GHz}$ dust emission in the Taurus-California-Perseus region, we conclude that the B211/B213 filament was probably formed as a result of the expansion of a large-scale supershell originated in the Per OB2 association. This scenario provides a simple explanation for the different inclinations of the northeastern and southwestern sheet components inferred from our modeling analysis.

6. Based on these results, we propose that (a) large-scale compression(s) generated by the Per OB2 association initially formed the B211/B213 filament system, and (b) accretion of ambient gas material due to the gravitational potential of the filament is now responsible for the growth of the filament.

Acknowledgements. This work was supported by the ANR-11-BS56-010 project "STARFICH" and the European Research Council under the European Union's Seventh Framework Programme (ERC Advanced Grant Agreement No. 291294 "ORISTARS"). Y.S, also received support from the ANR (project NIKA2SKY, grant agreement ANR-15-CE31-0017). P.P. acknowledges support from the Fundação para a Ciência e a Tecnologia of Portugal (FCT) through national funds (UID/FIS/04434/2013) and by FEDER through COMPETE2020 (POCI01-0145-FEDER-007672) and also by the fellowship SFRH/BPD/110176/2015 funded by FCT (Portugal) and POPH/FSE (EC). This research has made use of "Aladin sky atlas" developed at CDS, Strasbourg Observatory, France (Bonnarel et al. 2000; Boch \& Fernique 2014).

\section{References}

André, P., Men'shchikov, A., Bontemps, S., et al. 2010, A\&A, 518, L102 André, P., Di Francesco, J., Ward-Thompson, D., et al. 2014, Protostars and Planets VI (Tucson, AZ: University of Arizona Press), 27

Arzoumanian, D., André, P., Didelon, P., et al. 2011, A\&A, 529, L6

Arzoumanian, D., Shimajiri, Y., Inutsuka, S.-I., Inoue, T., \& Tachihara, K. 2018, PASJ, 70, 96

Arzoumanian, D., André, P., Könyves, V., et al. 2019, A\&A, 621, A42

Bally, J., Walawender, J., Johnstone, D., Kirk, H., \& Goodman, A. 2008, in Handbook of Star Forming Regions, ed. B. Reipurth, 308

Benedettini, M., Pezzuto, S., Schisano, E., et al. 2018, A\&A, 619, A52

Boch, T., \& Fernique, P. 2014, ASP Conf. Ser., 485, 277

Bonnarel, F., Fernique, P., Bienaymé, O., et al. 2000, A\&AS, 143, 33

Cernis, K. 1993, Balt. Astron., 2, 214

Chapman, N. L., Goldsmith, P. F., Pineda, J. L., et al. 2011, ApJ, 741, 21

Chen, C.-Y., \& Ostriker, E. C. 2014, ApJ, 785, 69

Chen, H., Gao, Y., Braine, J., \& Gu, Q. 2015, ApJ, 810, 140

Chen, C.-Y., Li, Z.-Y., King, P. K., \& Fissel, L. M. 2017, ApJ, 847, 140

Clarke, S. D., Whitworth, A. P., Duarte-Cabral, A., \& Hubber, D. A. 2017, MNRAS, 468, 2489

Dhabal, A., Mundy, L. G., Rizzo, M. J., Storm, S., \& Teuben, P. 2018, ApJ, 853, 169

Elias, J. H. 1978, ApJ, 224, 857

Finkbeiner, D. P. 2003, ApJS, 146, 407

Gao, Y., \& Solomon, P. M. 2004, ApJ, 606, 271

Goldsmith, P. F., Heyer, M., Narayanan, G., et al. 2008, ApJ, 680, 428

Hacar, A., Tafalla, M., Kauffmann, J., \& Kovács, A. 2013, A\&A, 554, A55

Heitsch, F. 2013, ApJ, 769, 115

Hennebelle, P., \& André, P. 2013, A\&A, 560, A68

HI4PI Collaboration (Ben Bekhti, N., et al.) 2016, A\&A, 594, A116

Inoue, T., Hennebelle, P., Fukui, Y., et al. 2018, PASJ, 70, S53

Inutsuka, S.-I., Inoue, T., Iwasaki, K., \& Hosokawa, T. 2015, A\&A, 580, A49

Kalberla, P. M. W., Kerp, J., Haud, U., \& Haverkorn, M. 2017, A\&A, 607, A15

Könyves, V., Kiss, C., Moór, A., Kiss, Z. T., \& Tóth, L. V. 2007, A\&A, 463, 1227

Könyves, V., André, P., Men'shchikov, A., et al. 2015, A\&A, 584, A91

Lada, C. J., Lombardi, M., \& Alves, J. F. 2010, ApJ, 724, 687

Lada, C. J., Forbrich, J., Lombardi, M., \& Alves, J. F. 2012, ApJ, 745, 190

Lallement, R., Vergely, J.-L., Valette, B., et al. 2014, A\&A, 561, A91

Langer, W. D., \& Penzias, A. A. 1993, ApJ, 408, 539

Li, D., \& Goldsmith, P. F. 2012, ApJ, 756, 12

Lim, T.-H., Min, K.-W., \& Seon, K.-I. 2013, ApJ, 765, 107

Marsh, K. A., Kirk, J. M., André, P., et al. 2016, MNRAS, 459, 342

Men'shchikov, A., André, P., Didelon, P., et al. 2010, A\&A, 518, L103

Narayanan, G., Heyer, M. H., Brunt, C., et al. 2008, ApJS, 177, 341

Olano, C. A., \& Poeppel, W. G. L. 1987, A\&A, 179, 202

Palmeirim, P., André, P., Kirk, J., et al. 2013, A\&A, 550, A38

Panopoulou, G. V., Tassis, K., Goldsmith, P. F., \& Heyer, M. H. 2014, MNRAS, 444, 2507

Planck Collaboration I. 2014, A\&A, 571, A1

Planck Collaboration Int. XXXIII. 2016, A\&A, 586, A136

Qian, L., Li, D., Offner, S., \& Pan, Z. 2015, ApJ, 811, 71

Seifried, D., \& Walch, S. 2015, MNRAS, 452, 2410

Sfeir, D. M., Lallement, R., Crifo, F., \& Welsh, B. Y. 1999, A\&A, 346, 785

Shimajiri, Y., André, P., Braine, J., et al. 2017, A\&A, 604, A74

Smith, M. D. 1994, ApJ, 421, 400

Smith, M. D. 2012, Astrophysical Jets and Beams (Cambridge: Cambridge University Press)

Snowden, S. L., Egger, R., Finkbeiner, D. P., Freyberg, M. J., \& Plucinsky, P. P. 1998, ApJ, 493, 715

Tafalla, M., \& Hacar, A. 2015, A\&A, 574, A104 


\section{Appendix A: Complementary figures}

Figure A.1 is a flowchart of our Gaussian fitting procedure with $N$ components. Figure A.2 shows the $12 \mathrm{CO}(1-0)$ and $13 \mathrm{CO}(1-0)$ velocity channel maps after subtracting the components that are not associated with the B211/B213 filament (see Sect. 4.2.1). Figure A.3 is a flowchart for our three-component modeling procedure described in Sect. 4.2.2. Figures A.4 and A.5 show the large-scale spatial distribution of $\mathrm{HI}$ emission in the Taurus-Auriga-California-Perseus region based on the HI data from Kalberla et al. (2017). Figure A.6 shows the large-scale spatial distributions of the $\mathrm{H} \alpha$ emission (Finkbeiner 2003) and $857 \mathrm{GHz}$ dust emission (Planck Collaboration I 2014; see also Fig. 10 and Sect. 4.3).

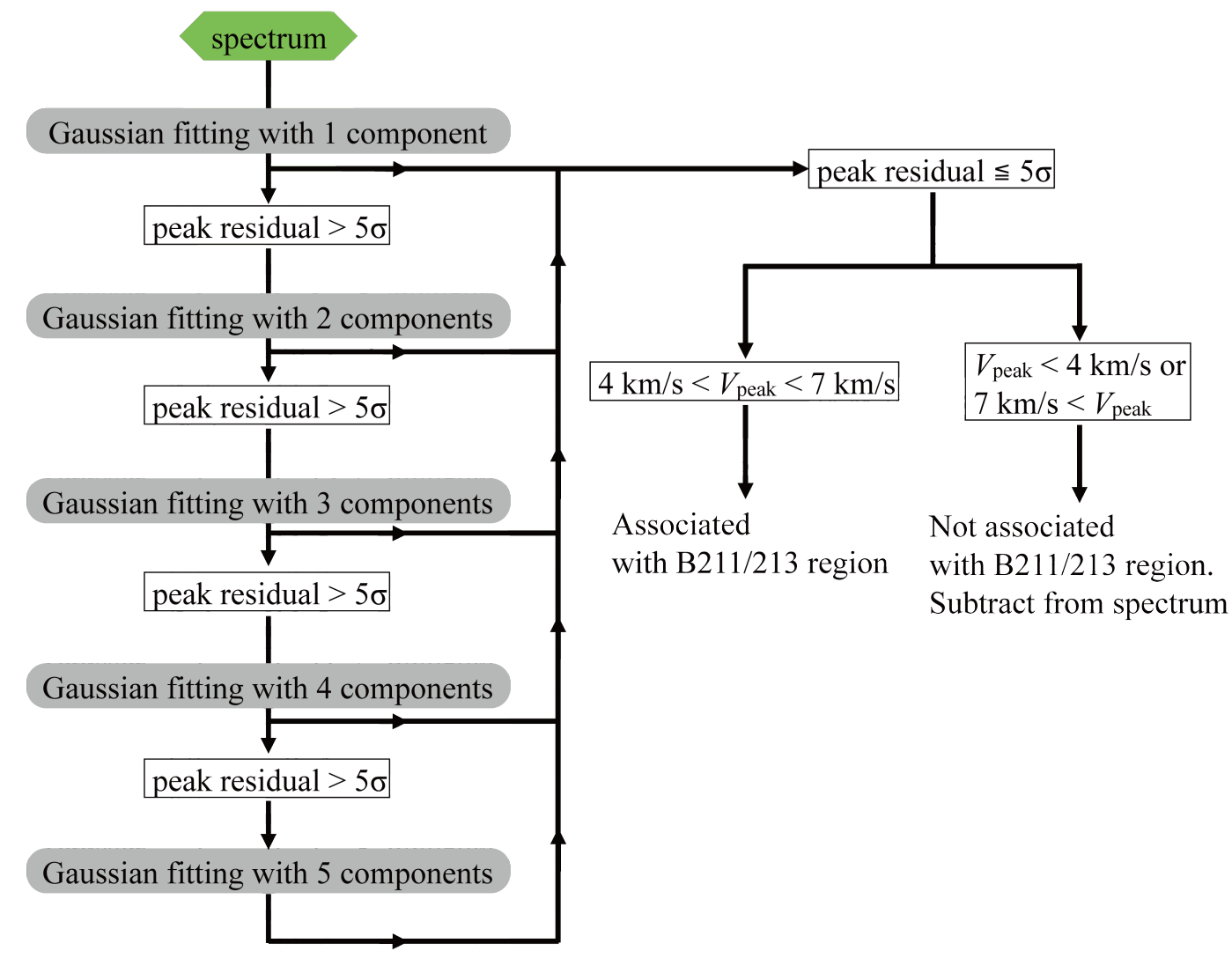

Fig. A.1. Flowchart of our Gaussian fitting procedure with $N$ components. Gaussian fitting with $N=1$ component is first applied to each pixel. Where the $\mathrm{S} / \mathrm{N}$ of the residual peak intensity is lower than 5 , we considered that the spectrum at this pixel consists of $N=1$ Gaussian component. When the $\mathrm{S} / \mathrm{N}$ of the residual peak intensity is higher than 5, we applied Gaussian fitting with $N+1$ Gaussian components. This process was repeated for five components at most. Then, when the peak velocity is lower than $4.0 \mathrm{~km} \mathrm{~s}^{-1}$ or higher than $7.0 \mathrm{~km} \mathrm{~s}^{-1}$, we considered that the component is not associated with the B211/B213 cloud and subtracted it from the data cube. 

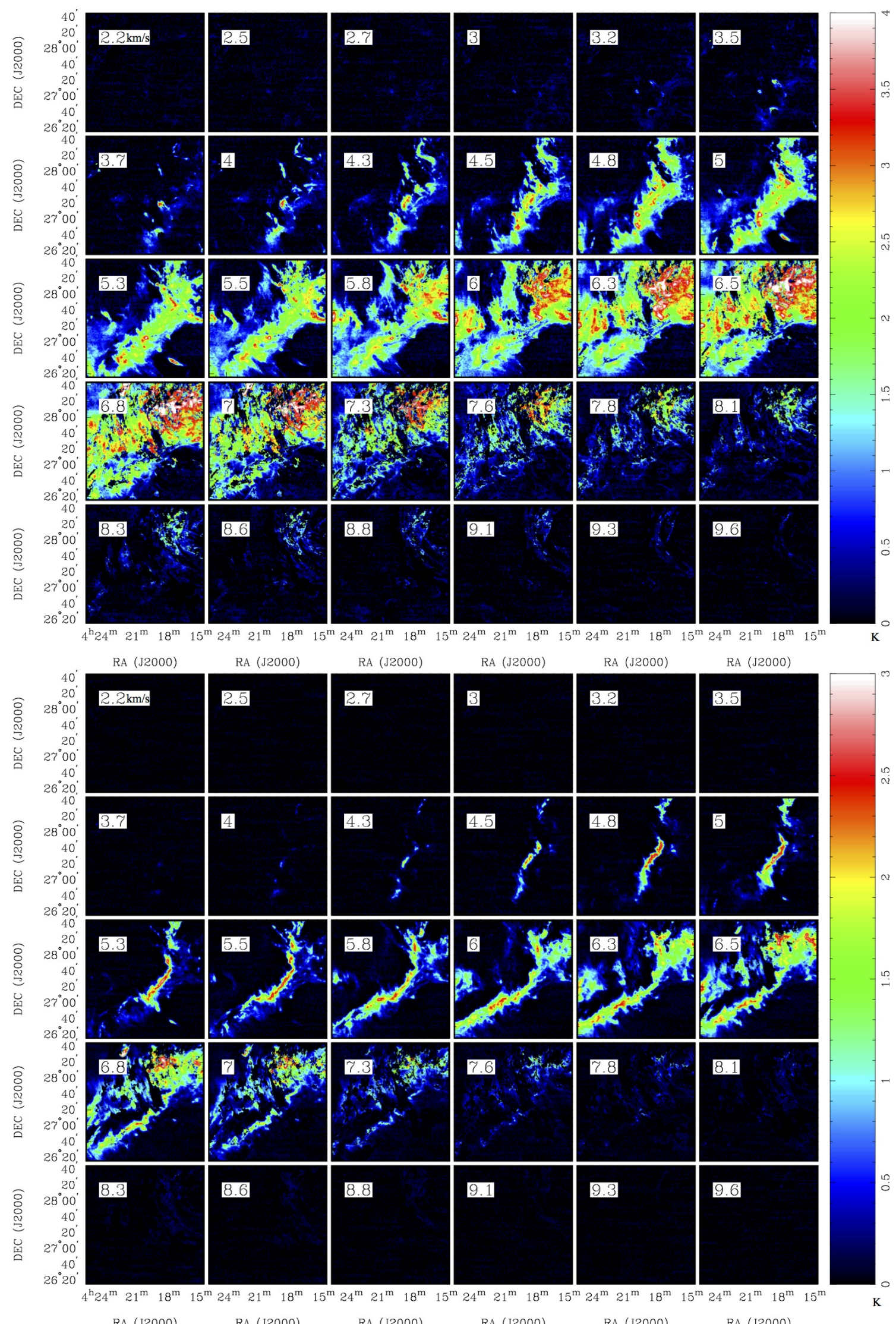

Fig. A.2. ${ }^{12} \mathrm{CO}(J=1-0$, top panel $)$ and ${ }^{13} \mathrm{CO}(J=1-0$, bottom panel $)$ velocity channel maps after subtracting the components that are not associated with the B211/B213 filament. The intensity unit is K. The velocities in $\mathrm{km} \mathrm{s}^{-1}$ are indicated in the top left corner of each panel. The velocity width of each channel map is $0.3 \mathrm{~km} \mathrm{~s}^{-1}$. 
Y. Shimajiri et al:: Accretion into the Taurus filament

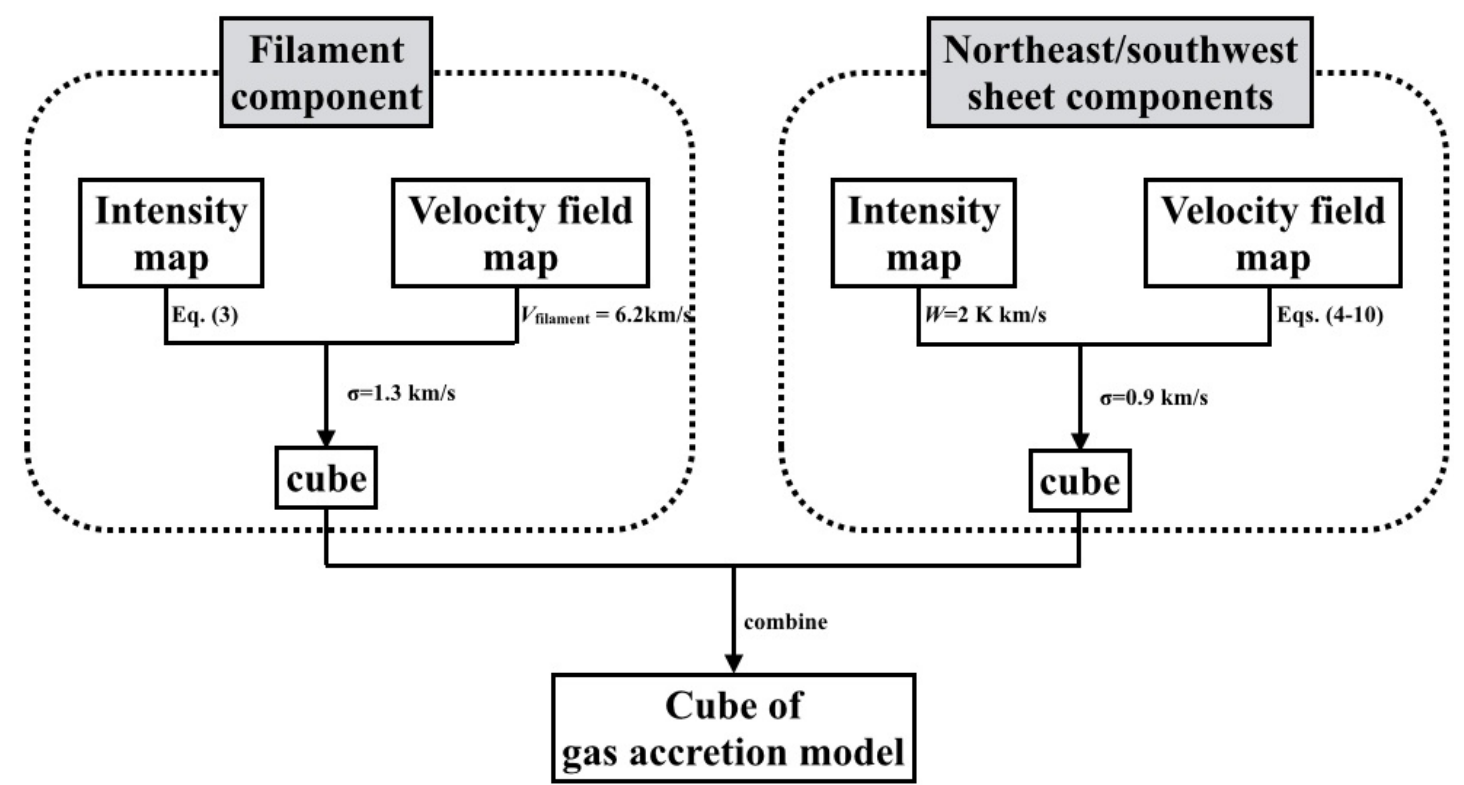

Fig. A.3. Flowchart for the three-component modeling procedure (see Sect. 4.2.2).

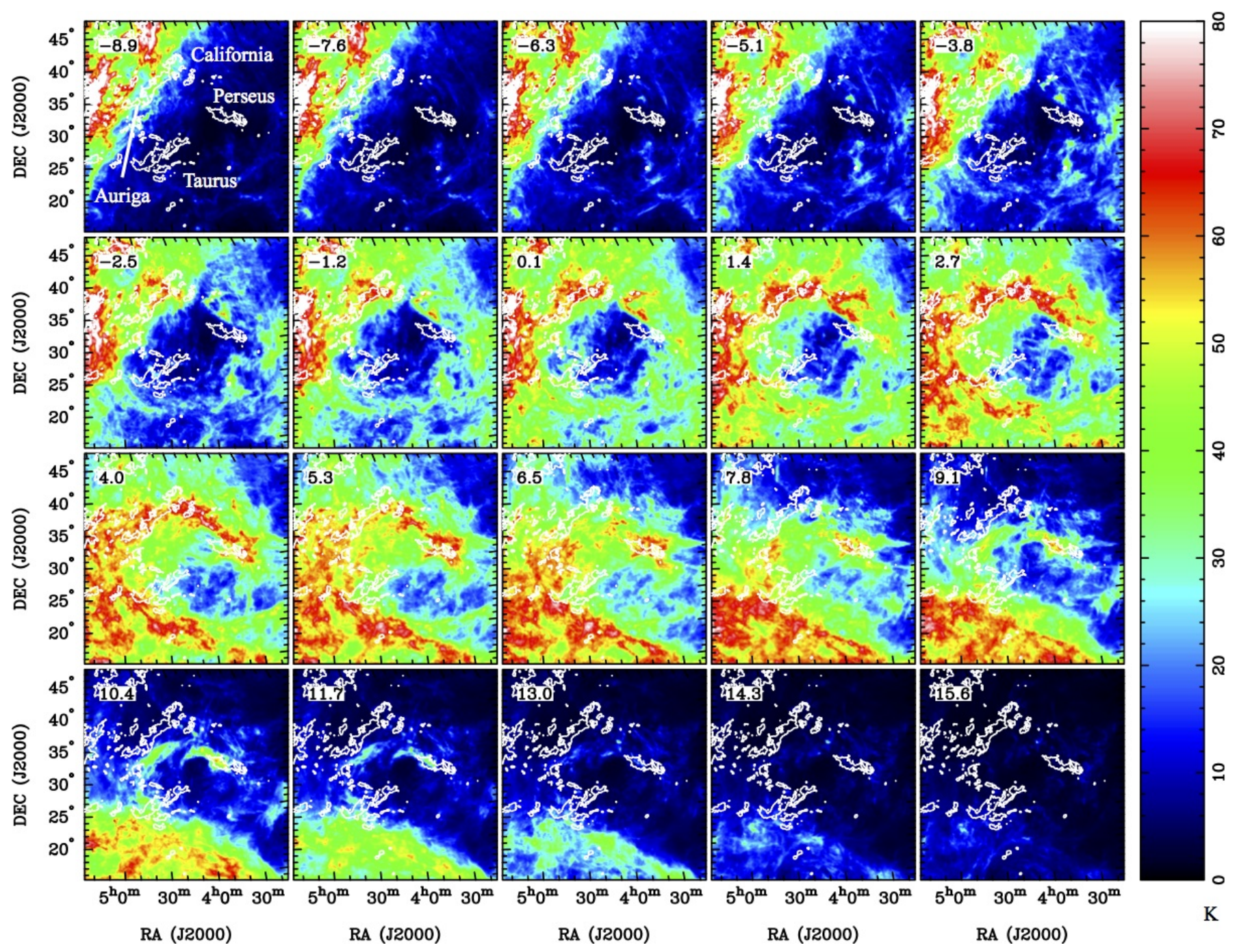

Fig. A.4. HI velocity channel maps. The HI data are from the Effelsberg-Bonn HI Survey (EBHIS) and Galactic All-Sky Survey (GASS; HI4PI Collaboration 2016). The intensity unit is $\mathrm{K}$. The contour indicates a level of $30 \mathrm{MJy} \mathrm{str}^{-1}$ at the Planck $857 \mathrm{GHz}$ emission. The velocities are indicated in the top left corner of each panel. The velocity width of each channel map is $1.3 \mathrm{~km} \mathrm{~s}^{-1}$. 


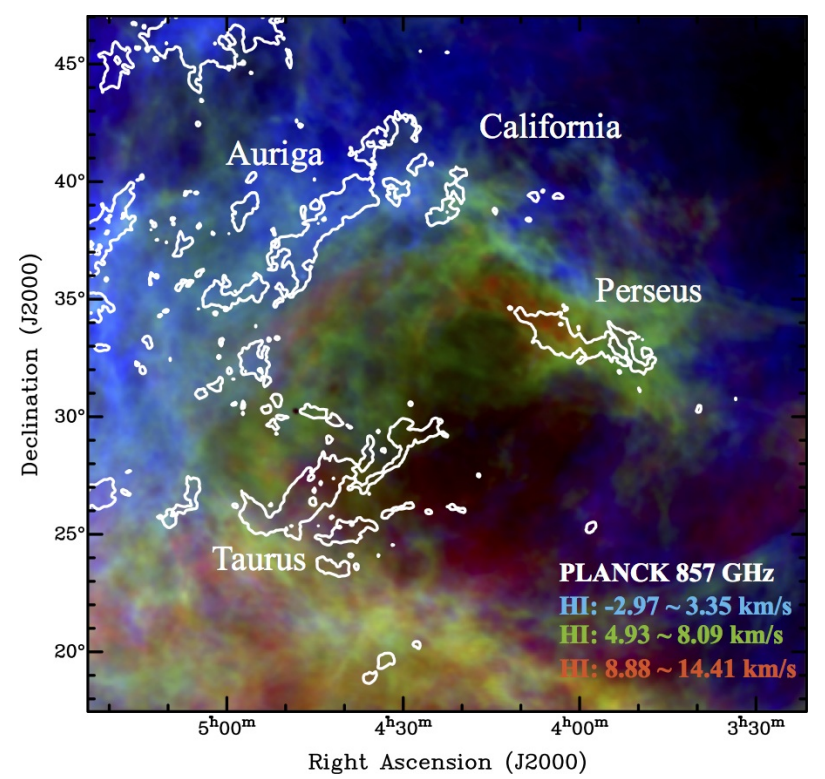

Fig. A.5. Large-scale spatial distribution of HI emission in the TaurusAuriga-California-Perseus region from Kalberla et al. (2017). Red, green, and blue are the HI components at the velocity of -29.7 to $3.35,4.93-8.09$, and $8.88-14.41 \mathrm{~km} \mathrm{~s}^{-1}$. The contour indicates a level of $30 \mathrm{MJy} \mathrm{str}^{-1}$ at the Planck $857 \mathrm{GHz}$ emission.
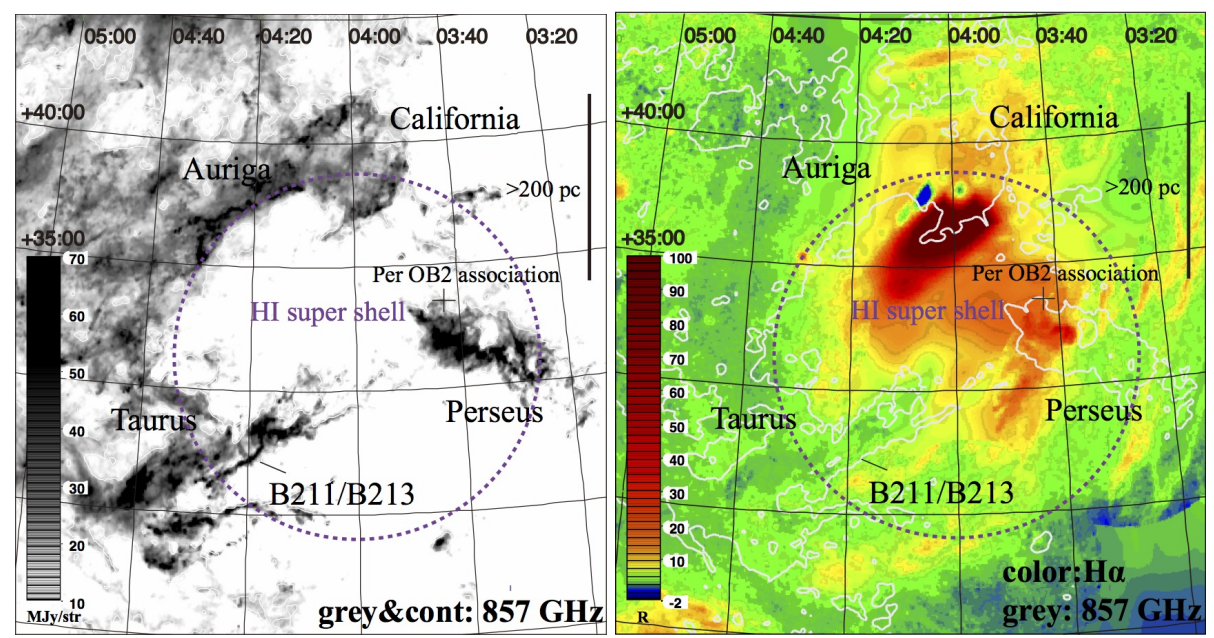

Fig. A.6. Spatial distributions of the $\mathrm{H} \alpha$ (color; left panel; Finkbeiner 2003) and $857 \mathrm{GHz}$ dust (gray; right panel; Planck Collaboration I 2014) emission. Same as Fig. 10, but the $\mathrm{H} \alpha$ and Planck $857 \mathrm{GHz}$ maps are displayed separately for clarity. The contour indicates a level of $10 \mathrm{MJy} \mathrm{str}^{-1}$ at the Planck $857 \mathrm{GHz}$ emission. 


\section{Appendix B: Inclinations of the two sheet components in the model}

To investigate the effect of the assumed inclinations for the two sheet components in our model, we expected a range of inclinations $\left(10^{\circ}, 20^{\circ}, 30^{\circ}, 40^{\circ}, 50^{\circ}, 60^{\circ}, 70^{\circ}\right.$, and $\left.80^{\circ}\right)$ and compared for each inclination the (peak) velocities predicted by the model with the ${ }^{12} \mathrm{CO} /{ }^{13} \mathrm{CO}$ observations. The northeastern and southwestern sheet components were examined separately. We assumed the same parameters as listed in Table 1, except for the inclination angles $\left(\theta_{\mathrm{N} / \mathrm{S}}\right)$. Figure B.1 shows the velocity offsets between models and observations. The velocity offset for the southwestern sheet component (offset $>0$ ) increases as the inclination angle increases, while the velocity offset for the northeastern sheet component (offset $<0$ ) increases as the inclination angle decreases. The velocity offset at loffsetl $<R_{\text {out }}$ tends to be larger than that at loffsetl $>R_{\text {out }}$. One possible reasons is that the ${ }^{12} \mathrm{CO}(1-0)$ and ${ }^{13} \mathrm{CO}(1-0)$ emissions do not trace the inner part of the filament (loffsetl $<R_{\text {out }}$ ) since the ${ }^{12} \mathrm{CO}(1-0)$ and ${ }^{13} \mathrm{CO}(1-0)$ optical depths are much larger than unity (see Sect. 3.1). In order to further investigate the velocity fields of the accreting gas, observations in optically thin dense gas tracers such as $\mathrm{N}_{2} \mathrm{H}^{+}(1-0)$ and $\mathrm{H}^{13} \mathrm{CO}^{+}(1-0)$, which trace the filament well (cf. Shimajiri et al. 2017, for $\mathrm{H}^{13} \mathrm{CO}^{+}(1-0)$ ), are required. Table B.1 summarizes the mean values of the velocity offsets for the northeastern and southwestern sheet components for each model. Inclinations of $70^{\circ}$ for the northeastern sheet component and $20^{\circ}$ for the southwestern sheet component provide the minimum velocity offset. We therefore adopted these inclination values in the model presented in Sect. 4.2.2.
Table B.1. Mean values of the velocity offset between ${ }^{12} \mathrm{CO}(1-0) /{ }^{13} \mathrm{CO}$ $(1-0)$ observations and model.

\begin{tabular}{|c|c|c|c|}
\hline Inclination $^{a}$ & North/South & Line & Mean \pm Std. dev. ${ }^{b}$ \\
\hline \multirow{4}{*}{$10^{\circ}$} & North & ${ }^{12} \mathrm{CO}$ & $0.48 \pm 0.44$ \\
\hline & South & ${ }^{12} \mathrm{CO}$ & $0.29 \pm 0.25$ \\
\hline & North & ${ }^{13} \mathrm{CO}$ & $0.41 \pm 0.49$ \\
\hline & South & ${ }^{13} \mathrm{CO}$ & $0.25 \pm 0.19$ \\
\hline \multirow{4}{*}{$20^{\circ}$} & North & ${ }^{12} \mathrm{CO}$ & $0.48 \pm 0.17$ \\
\hline & South & ${ }^{12} \mathrm{CO}$ & $0.26 \pm 0.24$ \\
\hline & North & ${ }^{13} \mathrm{CO}$ & $0.43 \pm 0.17$ \\
\hline & South & ${ }^{13} \mathrm{CO}$ & $0.17 \pm 0.15$ \\
\hline \multirow{4}{*}{$30^{\circ}$} & North & ${ }^{12} \mathrm{CO}$ & $0.50 \pm 0.14$ \\
\hline & South & ${ }^{12} \mathrm{CO}$ & $0.27 \pm 0.24$ \\
\hline & North & ${ }^{13} \mathrm{CO}$ & $0.45 \pm 0.17$ \\
\hline & South & ${ }^{13} \mathrm{CO}$ & $0.21 \pm 0.17$ \\
\hline \multirow{4}{*}{$40^{\circ}$} & North & ${ }^{12} \mathrm{CO}$ & $0.50 \pm 0.14$ \\
\hline & South & ${ }^{12} \mathrm{CO}$ & $0.26 \pm 0.19$ \\
\hline & North & ${ }^{13} \mathrm{CO}$ & $0.45 \pm 0.17$ \\
\hline & South & ${ }^{13} \mathrm{CO}$ & $0.25 \pm 0.19$ \\
\hline \multirow{4}{*}{$50^{\circ}$} & North & ${ }^{12} \mathrm{CO}$ & $0.50 \pm 0.14$ \\
\hline & South & ${ }^{12} \mathrm{CO}$ & $0.31 \pm 0.29$ \\
\hline & North & ${ }^{13} \mathrm{CO}$ & $0.45 \pm 0.17$ \\
\hline & South & ${ }^{13} \mathrm{CO}$ & $0.37 \pm 0.26$ \\
\hline \multirow{4}{*}{$60^{\circ}$} & North & ${ }^{12} \mathrm{CO}$ & $0.38 \pm 0.23$ \\
\hline & South & ${ }^{12} \mathrm{CO}$ & $0.47 \pm 0.41$ \\
\hline & North & ${ }^{13} \mathrm{CO}$ & $0.33 \pm 0.14$ \\
\hline & South & ${ }^{13} \mathrm{CO}$ & $0.54 \pm 0.44$ \\
\hline \multirow{4}{*}{$70^{\circ}$} & North & ${ }^{12} \mathrm{CO}$ & $0.26 \pm 0.28$ \\
\hline & South & ${ }^{12} \mathrm{CO}$ & $0.62 \pm 0.45$ \\
\hline & North & ${ }^{13} \mathrm{CO}$ & $0.20 \pm 0.18$ \\
\hline & South & ${ }^{13} \mathrm{CO}$ & $0.63 \pm 0.52$ \\
\hline \multirow{4}{*}{$80^{\circ}$} & North & ${ }^{12} \mathrm{CO}$ & $0.29 \pm 0.35$ \\
\hline & South & ${ }^{12} \mathrm{CO}$ & $0.73 \pm 0.51$ \\
\hline & North & ${ }^{13} \mathrm{CO}$ & $0.21 \pm 0.29$ \\
\hline & South & ${ }^{13} \mathrm{CO}$ & $0.74 \pm 0.60$ \\
\hline
\end{tabular}

Notes. ${ }^{(a)}$ Assumed inclinations for the northeast and southwest sheet components in the model. ${ }^{(b)}$ Mean and standard deviation of the velocity offset between observations and model. 

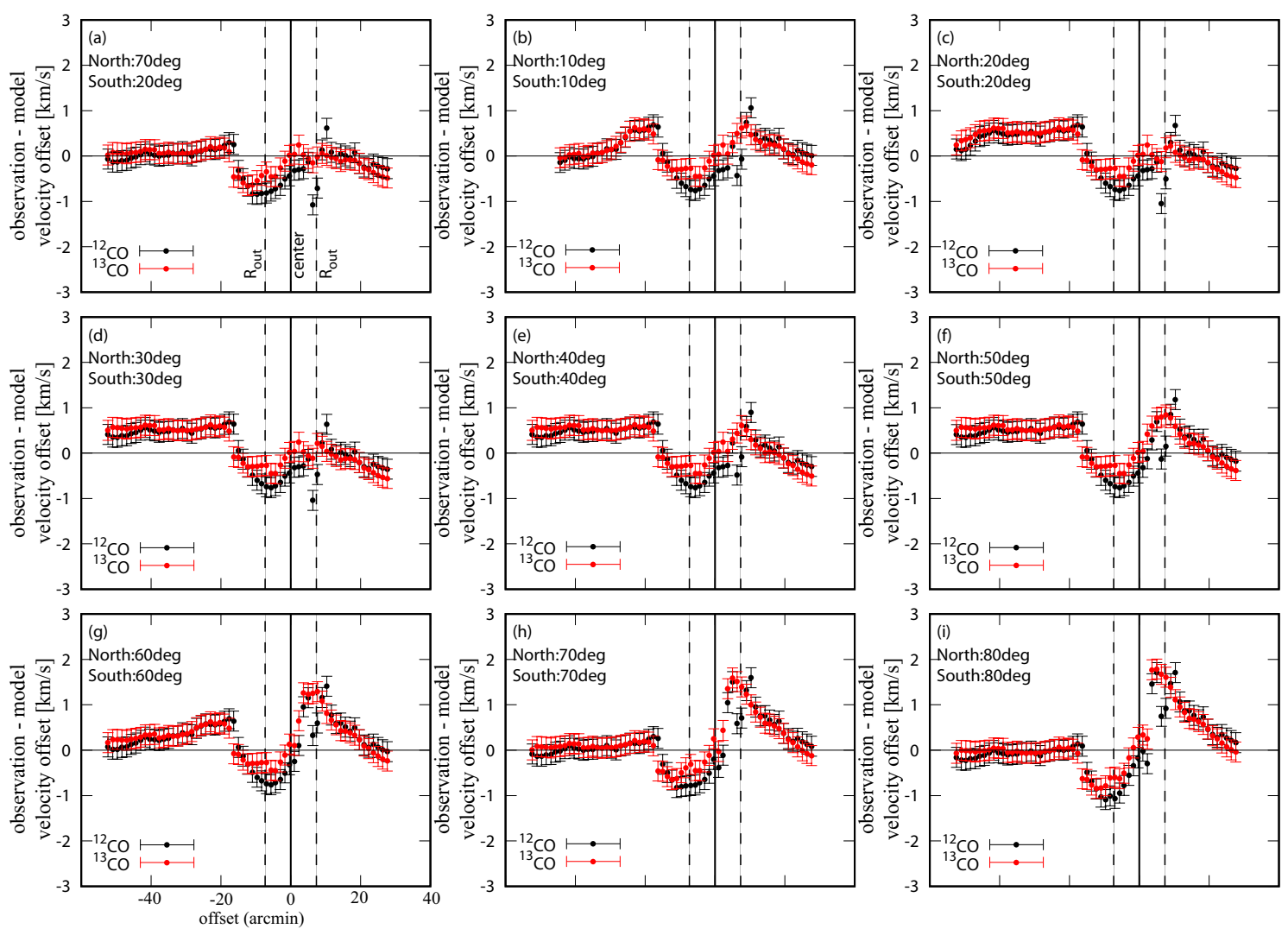

Fig. B.1. Velocity offset as a function of position between the model and ${ }^{12} \mathrm{CO}(1-0) /{ }^{13} \mathrm{CO}(1-0)$ in the $P V$ diagrams. The assumed inclination angles for the sheet in the model are indicated in the top left corner of each panel. Black and red indicate the velocity differences between the model and ${ }^{12} \mathrm{CO}(1-0)$ data and between the model and ${ }^{13} \mathrm{CO}(1-0)$ data, respectively. 